\title{
Combined Effects of Nanoroughness and lons Produced by Electrodeposition of Mesoporous Bioglass Nanoparticle for Bone Regeneration
}


Singh ${ }^{1,2}$, Jonathan C. Knowles ${ }^{2,4,5,6}$, Hae-Won Kim ${ }^{1,2,3,4}$

${ }^{1}$ Institute of Tissue Regeneration Engineering (ITREN), Dankook University, Cheonan 31116, South Korea

2 Department of Nanobiomedical Science \& BK21 PLUS NBM Global Research Center for Regenerative Medicine, Dankook University, Cheonan 31116, South Korea

${ }^{3}$ Department of Biomaterials Science, School of Dentistry, Dankook University, Cheonan 31116, South Korea

${ }^{4}$ UCL Eastman-Korea Dental Medicine Innovation Centre, Dankook University, Cheonan 31116, Republic of Korea

${ }^{5}$ Division of Biomaterials and Tissue Engineering, Eastman Dental Institute, University College London, 256 Gray's Inn Road, London WC1X 8LD, United Kingdom

${ }^{6}$ The Discoveries Centre for Regenerative and Precision Medicine, UCL Campus, London, UK

*Corresponding author: Dr. K. D. Patel, tel: +82 41550 3081; fax: +82 415503085

E-mail: kapildpatel20@gmail.com, kapil.patel@ucl.ac.uk

For: ACS Applied Bio Materials 


\section{ABSTRACT}

Providing appropriate biophysical and biochemical cues to the interface is a facile strategy to enhance the osteogenic ability of metallic implants. Here we exploited this through the incorporation of mesoporous bioactive glass nanoparticles (MBGN) at a high content (1:1 by weight) to a biopolymer chitosan in the electrodeposition process of titanium. The MGBN/chitosan layer thickness, tunable by electrodeposition parameters, exhibited an accelerated ability of apatite mineral induction in a body simulating medium. Of note, the involvement of MBGN could generate nanoscale roughness in a unique range of $10 \sim 25 \mathrm{~nm}$. Moreover, the layer showed a slowly releasing profile of ions (calcium and silicate) over weeks at therapeutically relevant doses. The ion-releasing nanotopological surface was demonstrated to alter the pre-osteoblasts responses in a way favorable for the osteogenic differentiation. The combinatory cues of nanotopology ( $25 \mathrm{~nm}$ roughness) and ion release enabled highly accelerated cellular anchorage with somewhat limited spreading area at initial periods. The subsequent osteoblastic differentiation behaviors on the engineered surface, as examined up to 21 days, showed significantly enhanced alkaline phosphate activity and up-regulated expression of bone-associated genes (ALP, Col I, OPN, and OCN). These results indicate that the combinatory cues provided by nanotopology ( $25 \mathrm{~nm}$ roughness) and ions released from MBGN are highly effective in stimulating osteoblastic differentiation and that suggest the MBGN/chitosan may serve as a potential composition for bone implant coatings.

Keywords: Mesoporous bioglass coatings; Nano/microstructure; Electro-deposition; Cell adhesion; Osteoblast activity 


\section{INTRODUCTION}

Metallic implant biomaterials are design and developed to replaces hard tissue such as artificial hip joints, bone plates, and dental implants ${ }^{1}$. Pure titanium and its alloys are the frequently used metallic implants due to their suitable combination mechanical, biocompatibility, and electrochemical properties. The necessity to fabricate metal implant devices to clinically treat to bone disorders and bone disease with long-term stability remains a clinical challenge. Bone disorders are very common and occurs in 124 out of 1000 person in the USA causing long-term pain and physical disability ${ }^{2}$. Although, titanium based metallic implants have gain huge acceptance in clinics, implant failure can occur specially in patients suffering from a thin gingival biotype ${ }^{3}$. Therefore, there is a need to improve the surface of the implant to develop bioatictve and biocompatible for smooth and easy osseointegration. A number of in vitro and in vivo studies showed that the implant with micro-/nanotopography surface, bioactive-chemical functionalization, and wettability regulate the cellular fate towards osteogenic and their progeny, ultimately determining the osseointegration potential and clinical success of an metallic implants ${ }^{4}$.

Physico-chemical properties of an implant including, surface chemistry, wettability, nanotopography, mechanical stability and bioactivity govern the long-term success of the medical implants through diverse cellular reactions ${ }^{5-6}$. Surface properties such as surface topography, chemistry and roughness have been thoroughly investigated and shown the huge impact in rapid bone integration with the implant. The wettability of the implant surface determines the consequent events during material-bone tissue integration. Moreover, wettability of implant surface can be controlled by appropriate modulation of surface topography and chemistry ${ }^{7}$. However, the current trend to design the implant surfaces focuses mainly on specific structural features, particularly either micro-/ nanotopography or combination of both, which have a suboptimal ability to promote rapid integration with the surrounding host tissues. There is increasing interest among researchers to develop tailored surface topography, chemistry and functionalization for the understanding of biological responses at the implant/bone or implant/soft tissue interface. Therefore, several coating techniques have been developed to produce tailored implant surface modification with controlled surface chemistry and topography and excellent biological function in vitro and in vivo ${ }^{8-9}$. 
The recent trend for implant surface modification is changing and shifted more towards including nanotopological features for better cell-material interaction and subsequent improvement in osseointegration. In this regard, several bioactive nanoparticles including nano-sized hydroxyapatite, calcium phosphates, bioglass nanoparticles, silica nanoparticles, calcium silicate-reduced graphene oxide and carbon nanotubes are utilized for implant surface modifications ${ }^{10-11}$. These nanomaterials can be used to produce microstructured and nanostructured coatings via various coating techniques ${ }^{12}$. The nanotopological surface generated by inclusion of micro/nanoparticles acts as a physical cue that can regulate and dictate the stem cell behavior ${ }^{13-14}$. Most of the cell-material interaction events are controlled through cellular sensing of extracellular nanotopological cues via nanoscale surface and initiate the intracellular mechanotransduction events ${ }^{15-16}$. Owing to the nanotopological features of composite coatings to stem cell regulation and therapeutic delivery, biomedical scientist and engineers are trying to achieve stem cell fate controlled for specific lineage through nanotopological surface technologies and materials processing methods ${ }^{17}$.

Mechanical stability and bioactivity of the coating are also important properties for the success of an implant. The mechanical strength of the coating is a result of optimization of composition and structural material. The compositions have an important role to provide essential physical, mechanical, biocompatibility, and cellular supporting microenvironments for long-term anchoring of the implants. In particular, the highly mesoporous bioactive glass nanoparticles can significantly affect the coating surface integrity including, surface topography, roughness, hardness, wettability and subsequently biocompatibility. Moreover, the nanotopological feature of the coatings not only provides huge advantages for excellent cell-material interactions but also allows biomolecules adsorption due to the high surface area ${ }^{18}$. Furthermore, nanosized MBGN coatings surface used in bone regeneration applications has shown to emulate the biochemical and biomechanical (mechanotransduction) properties in the osseointegration process ${ }^{19-20}$.

Bioglass nanoparticles are well established inorganic nanomaterials used for various biomedical applications including drug delivery ${ }^{21}$, wound healing ${ }^{22}$, osteochondral regeneration ${ }^{23}$, and cell engineering applications ${ }^{24}$. Particularly, MBGN are excellent bone-bonding inorganic nanomaterials, which improve bioactivity and fixation by stimulating bone formation starting from the micro- 
nanotopological implant surface ${ }^{25}$. Therefore, MBGN are applied as a coating on mechanically strong titanium metal surfaces for bone regeneration.

In this study, we designed a nanostructured bioactive coating made of MBGN integrated with chitosan laid down via the electrophoretic deposition (EPD) method. The inorganic-organic composite coating conditions are controlled to prepare a surface merited from the nanotopological, and bioactive properties of MBGN therapeutic ions ( $\mathrm{Ca}, \mathrm{Si})$. The mechanical strength and nanoroughness of the composite coatings was analyzed by nanoindentation and attached scanning probe microscopy (SPM), respectively. Finally, the in vitro bone-bioactivity and the osteoblastic cell responses on the coatings were examined to find suitable applications for hard tissue implants. The scheme of the current study utilizing EPD for MBGN-Chi composite for bone regeneration is illustrated in Figure 1.

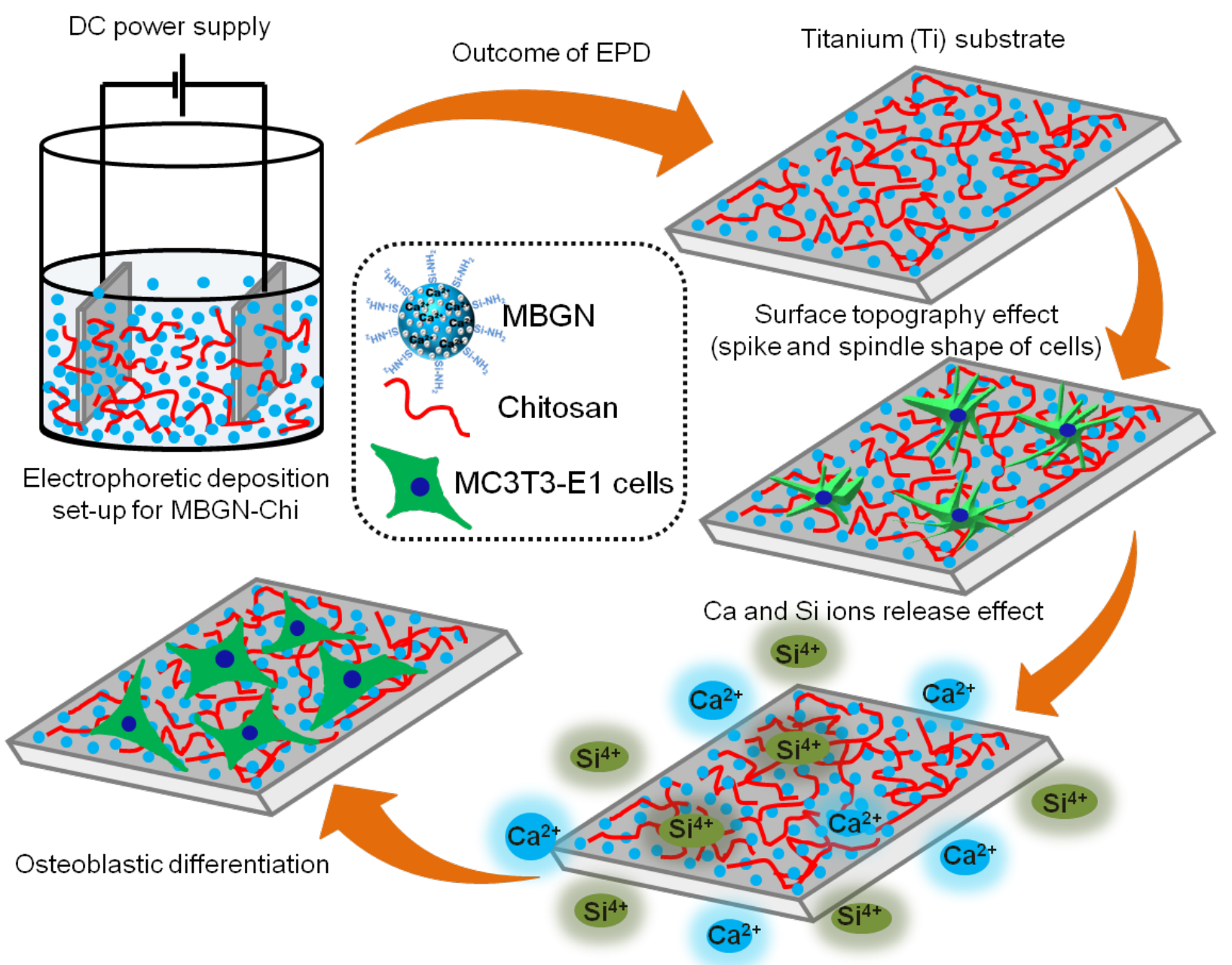


Figure 1. Schematic illustration of EPD of MBGN/Chi and its role in promoting osteogenic differentiation. The EPD set-up for MBGN-chitosan and the outcome of EPD as bioactive nano-coating generates the nano-biointerface. The surface topography influenced the cell morphologies and further calcium and silicate ions released from the coatings and the osteogenic differentiation of MC3T3-E1 cells at the nanobiointerface as combined effect of nanotopography plus ions release.

\section{EXPERIMENTAL SECTION}

\subsection{Materials}

Commercially pure titanium (Ti; Senulbio Biotech, Korea) in rectangular plate form $(10 \mathrm{~mm} \times 10 \mathrm{~mm} \times 1$ $\mathrm{mm}$ ) was used for the coatings. Chitosan (Chi) of medium molecular weight (MW=200,000 Da, degree of deacetylation $\sim 85 \%$ ), silicon carbide sand paper (CC-2000 Cw, Daesung, Korea), acetic acid ( $\geq 99 \%$ ), hexadecyltrimethylammonium bromide $(\mathrm{CTAB})$, ethyl ether $\left(\mathrm{C}_{4} \mathrm{H}_{10} \mathrm{O}, 99 \%\right), \mathrm{Ca}\left(\mathrm{NO}_{3}\right)_{2} .4 \mathrm{H}_{2} \mathrm{O}, \mathrm{NH}_{4} \mathrm{OH}$ (28\% $\mathrm{NH}_{3}$ in water, $\geq 99.99 \%$ metal basis), tetraethyl orthosilicate (TEOS, $\mathrm{C}_{8} \mathrm{H}_{20} \mathrm{O}_{4} \mathrm{Si}, 98 \%$ ), methanol anhydrous $\left(\mathrm{CH}_{4} \mathrm{O}, 99.8 \%\right)$, toluene anhydrous $\left(\mathrm{C}_{7} \mathrm{H}_{8}, 99.8 \%\right)$, and 3-aminopropyl triethoxysilane (APTES, $\mathrm{C}_{9} \mathrm{H}_{23} \mathrm{NO}_{3} \mathrm{Si}$, $\geq 98 \%$ ) were purchased from Sigma-Aldrich and used as-received without any further purification.

\subsection{Mesoporous Bioactive Glass Nanoparticles Preparation and Amine Functionalization}

Mesoporous bioactive glass nanoparticles (MBGN) were prepared as previously described ${ }^{26}$. Briefly, in an emulsion system composed of $1 \mathrm{~g} \mathrm{CTAB}$ dissolved in $150 \mathrm{~mL}$ of $\mathrm{H}_{2} \mathrm{O}, 40 \mathrm{~mL}$ of ethyl ether, $20 \mathrm{~mL}$ of ethanol, $2 \mathrm{~mL}$ of aqueous ammonia, and calcium nitrate tetrahydrate $\left(\mathrm{Ca}\left(\mathrm{NO}_{3}\right)_{2} \cdot 4 \mathrm{H}_{2} \mathrm{O}\right)$. Then the solution was stirred at $25{ }^{\circ} \mathrm{C}$ for $30 \mathrm{~min}$, tetraethyl orthosilicate (TEOS) was quickly mixed into the solution. The mesoporous bioglass nanoparticles possessed a molar ratio of $\mathrm{Ca} / \mathrm{Si}$ is $15 / 85$. After $4 \mathrm{~h}$ of vigorously stirred at $25^{\circ} \mathrm{C}$, white precipitate was obtained by centrifugation and washed with distilled water, and then dried in air at $60{ }^{\circ} \mathrm{C}$ for $24 \mathrm{~h}$ and calcined at $600{ }^{\circ} \mathrm{C}$ for $6 \mathrm{~h}$ to remove any remaining CTAB. Further, the amine-functionalized of MBGN was carried out in toluene using (3-aminopropyl) triethoxysilane (APTES) by dispersing $0.1 \mathrm{~g}$ of MBGN in $50 \mathrm{~mL}$ of toluene and dispersed to a homogeneous solution. The solution and then refluxed at $80{ }^{\circ} \mathrm{C}$ for $24 \mathrm{~h}$, which was followed by a centrifugation at $8000 \mathrm{rpm}$ for $5 \mathrm{~min}$. The 
product was washed with absolute ethanol and dried overnight at $80^{\circ} \mathrm{C}$. Obtained MBGN nanoparticles were used for further characterizations and subsequent electrophoretic deposition.

\subsection{Characterizations of MBGN}

The particle size, shape and surface morphology of MBGN was carried out using field emission scanning electron microscope (FE-SEM; ZEISS Sigma 500, Germany) and high resolution transmission electron microscopy (HR-TEM; JEOL-7100, Japan) at 15 and 200 KV, respectively. X-ray diffraction (XRD; Rigaku

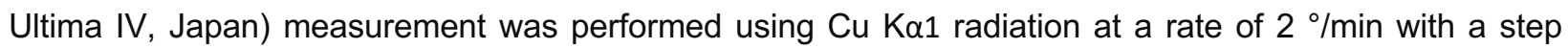
size 0.02 degree $2 \theta$ at $40 \mathrm{kV}$ and $40 \mathrm{~mA}$. Attenuated total reflectance Fourier transformed infrared spectroscopy (ATR-FTIR, Varian 640-IR, Australia) was performed for 25 scans with $4 \mathrm{~cm}^{-1}$ resolution to analyse the surface chemistry of MBGN and coating samples. The surface electrical potential of the

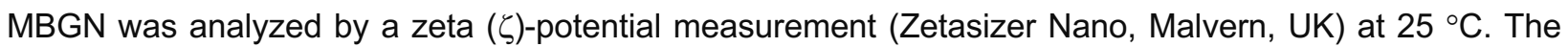
$\mathrm{N}_{2}$ adsorption /desorption isotherm were recorded on a Quantachrome automated sorption analyzer (Quantachrome; 1SI-MP-9, Germany). Obtained isotherms were analyzed using Barrett-Joyener-Halenda (BJH) for pore size and Brunar-Emmett-Teller (BTE) method for the specific surface area of the MBGN.

\subsection{Suspension for EPD}

For the EPD of chitosan and MBGN-Chi, it is important to select the optimum concentration of chitosan. A $200 \mathrm{~mL}$ stock solution of Chi with a concentration of $1 \mathrm{mg} / \mathrm{mL}$ was prepared in $1 \%$ acetic water-ethanol (75:25) solution by magnetic stirring. After complete dissolution of Chi, a clear transparent solution was obtained. Further, for the experiment Chi solution was diluted to $0.5 \mathrm{mg} / \mathrm{mL}$ by addition of $25 \%$ ethanol and maintain the $\mathrm{pH}$ of the suspension at 3.4 3.6. Next, varying amount of amine surface modified MBGN was added to $60 \mathrm{~mL}$ of $0.5 \mathrm{mg} / \mathrm{mL}$ Chi solution and sonicated to completely disperse the nanoparticles. The temperature of the sonication water bath was maintained with ice. After complete dispersion of MBGN, the resulting solution was used for the EPD process.

\subsection{EPD of Chi and MBGN-Chi}

Prior to start of the EPD process, titanium plates were polished on silicon carbide sandpaper (CC-2000 $\mathrm{Cw}$ ) and washed with distilled water under sonication, followed by ethanol and acetone, respectively. 
During the EPD process, the titanium plate was used as the cathode and stainless steel as the anode. The concentration of MBGN used was $0.25,0.50$, and $0.75 \mathrm{mg} / \mathrm{mL}$ while the chitosan concentration was maintained at $0.50 \mathrm{mg} / \mathrm{mL}$, the corresponding coating was named as MBGN-0.25, MBGN-0.50, and MBGN-0.75. The applied voltage and deposition time was optimized by trial-and-error method for uniform coating. Thus, for varying concentrating of MBGN the EPD of Chi and MBGN-Chi at $25 \mathrm{~V}$ and 4 minute was the optimum applied voltage and deposition time for homogeneous and uniform coatings. Electrode separation was maintained at $10 \mathrm{~mm}$ throughout the EPD process. After coatings, samples were dried at ambient temperature. Ethanol was used during the solution preparation to avoid any kind of hydrogen generation and bubble formation to affect the coating quality.

\subsection{Characterization of EPD Coatings}

Electrodeposited Chi and MBGN-Chi (i.e. MBGN-025, MBGN-0.50, and MBGN-0.75) composite coatings was investigated using scanning electron microscopy. The SEM images were taken after platinum sputter coating. The crystal phase of titanium and the presence of MBGN in the coatings was analyzed by XRD. The attenuated total reflectance Fourier-transform infrared spectroscopy was performed to characterize the Chi and MBGN-Chi coatings. Transmittance spectra were obtained in attenuated total reflection (ATR) mode and recorded between $400-4000 \mathrm{~cm}^{-1}\left(4 \mathrm{~cm}^{-1}\right.$ spectral resolution with scan rate of $\left.16 \mathrm{~Hz}\right)$. The amine surface modification of MBGN and chemicals bonds was also confirmed and studies by FT-IR. Thermogravimetric analysis (TGA; N-1500; Scinco, South Korea) of the coatings was carried out using portion of the coating layer scraped from the titanium surface under the nitrogen gas operation at temperature up to $600{ }^{\circ} \mathrm{C}$ at a heating rate of $10^{\circ} \mathrm{C} / \mathrm{min}$. Based on the TGA analysis, the quantity of the MBGN presence in the coating was determined.

\subsection{Physico-chemical Characterization of Coatings}

In-vitro apatite formation ability and bioactivity of the coatings was observed in $1 \mathrm{X}$ simulated body fluid (SBF) solution at $37^{\circ} \mathrm{C}$ in a water bath. Each coated sample (dimension of $10 \mathrm{~mm} \times 10 \mathrm{~mm} \times 1 \mathrm{~mm}$ ) was filled with $15 \mathrm{~mL}$ of SBF solution in a glass vial of $20 \mathrm{~mL}$ volume and incubated at $37^{\circ} \mathrm{C}$ for different time points. At each time point sample was removed, washed with distilled water and dried at room 
temperature. Further, samples were used for surface morphology observation and other characterizations.

\subsection{Mechanical Strength and Nanoroughness by Nanoindentation}

The mechanical strength and nanoroughness test was performed by nanoindentation (Hysitron TI 750, USA). The mechanical strength in terms of hardness $(H)$, elastic modulus $(E)$ and nanoroughness $(R a)$ of the EPD coated Chi and various MBGN-Chi coatings were analyzed by nanoindentation. The Nano module allow the application of a low target range from $500 \mu \mathrm{N}$ to $3 \mathrm{mN}$ ), using a piezo driver while monitoring the load with independent load cells and accurate recording of indenter position in the material with an independent capacitive depth sensor. The indentation parameter was optimized by the widely adopted $10 \%$ rule ${ }^{27}$. Briefly, to avoid any interference of substrate (titanium) properties with coatings, indentation depth should no more than $10 \%$ of total coating thickness. We first carried out the load vs. depth curve using a three sided pyramid (Berkovich indenters) tip for hardness and elastic modulus measurements. The Chi and MBGN-Chi coating surface through testing conditions: maximum load $3 \mathrm{mN}$; indentation depth from 500-3000 nm; and loading and unloading rate 100, 200, $400 \mu \mathrm{N} / \mathrm{s}$ for 500, 1000, and $3000 \mu \mathrm{N}$, respectively. We have also performed the same test with different maximum loadings (500 $\mu \mathrm{N}, 1000 \mu \mathrm{N}$, and $3000 \mu \mathrm{N}$ ) to identify the depth boundary for an independent measurement of coatings mechanical properties.

To evaluate both the nanoroughness properties and distribution effects (e.g., the uniformity distribution of the MBGN in the coatings), and taking into account the morphological differences between the coatings, maximum load $(3 \mathrm{mN})$ was tested to generate indent profile image using SPM attached to the nanoindentation. The indentation was made at various locations on the coating surface to provide a statistical sampling.

\subsection{Cell Viability Assay}

The cell viability of all coating samples was measured using the cell counting kit (CCK-8, Dojindo, Japan) that measure the accumulative metabolic activity. Briefly, the CCK solution was prepared by mixing CCK with culture media at a ratio of 1:10 (v/v). Three samples were treated for each incubation period $(3$, and 7 days). For every incubation period, the culture media was removed and samples were transferred to 
new 24 - well plates and filled with $500 \mu \mathrm{L}$ CCK solution in each well. After incubation for $3 \mathrm{~h}, 100 \mu \mathrm{L}$ of CCK-media solution from each well was transferred to 96 - well plate for optical density measurement using microplate reader at $450 \mathrm{~nm}$.

\subsection{Cell Adhesion, Spreading and Morphology}

Pre-osteoblastic MC3T3-E1 cells were cultured on Chi and MBGN-0.50 composite coatings, and then the cell morphology and proliferation were assessed for 7 days. Morphological analysis of pre-osteoblast cells seeded on both the samples was undertaken using SEM after 3 and 7 days. Briefly, after prescribed time periods, sample were removed from the culture plate and transferred to new well plates and then washed gently with PBS at least three times and fixed with $4 \%$ paraformaldehyde (PFA) for 5 min at room temperature. After fixation, samples were washed with PBS three times again and subsequently dehydrated in a graded series of ethanol $(70 \%, 80 \%, 95 \%$ and $100 \%)$ three times in each case for 5 min at room temperature. A $500 \mu \mathrm{L}$ of 1.1.1.3.3.3. hexamethyldisilazane (HMDS) solution was filled and samples were dried overnight in hood.

The morphology of adherent MC3T3-E1 cells on the chitosan and MBGN-0.50 nanotopographical composite coatings specimen was observed using scanning electron microscopy instrument at 10 and 15 $\mathrm{kV}$ acceleration voltage, respectively. The samples were sputter coated with platinum prior to examination of SEM. The cell morphologies were also observed using fluorescence microscope. Furthermore, initial cell adhesion and spreading were also analyzed from fluorescence images. The cell adhesion number was calculated by DAPI staining of cell nuclei image using ImageJ software at $24 \mathrm{~h}$ of cell culture. Next, phalloidin stained images were also applied for cell spreading area calculation using ImageJ software.

\subsection{Alkaline Phosphate Activity Assay}

The MC3T3-E1 cells were cultured on the Chi and MBGN-Chi modified titanium samples at a cell density of $1.0 \times 10^{4}$ cells $/ \mathrm{mL}$ to each sample to evaluate the alkaline phosphatase (ALP) activity. At each time point, the cells were dissociated from the surface and ALP enzymatic activity was assessed using ALP activity kit (Acid Phosphatase Activity Fluorometric Assay Kit, catalog \# MAK087, Sigma, USA). The double-stranded DNA (dsDNA) quantification was performed using the PicoGreen (Quant-iT тм 
PicoGreen $^{\mathrm{TM}}$ dsDNA Assay Kit) assay. The ALP activity was then normalized to the DNA content and the experiments were carried out in triplicates.

\subsection{Ions Release Test}

The ions (calcium, silicate) release profile from the coating was recorded over 21 days using inductively coupled plasma-atomic emission spectroscopy (ICP-AES; OPTIMA 4300 DV, Perkin-Elmer, USA). Briefly, MBGN-0.50 samples were placed in 24 well plates in $1 \mathrm{~mL}$ of Tris-buffer solution, $\mathrm{pH} 7.4$, and transferred inside water bath operated at $37^{\circ} \mathrm{C}$. At different time points, samples were taken and supernatants solution was centrifuged at 12,000 rpm for 15 min and collected for ion analysis.

\subsection{Osteogenic Differentiation}

The osteogenic differentiation of the MC3T3-E1 cells on Chi and MBGN-0.50 coatings were performed under culturing in osteogenic medium (minimum essential medium ( $\alpha-M E M), 1 \%$ penicillin (PS), $10 \mathrm{mM} \beta$ glycerophosphate ( $\beta$-GP), $10 \mathrm{nM}$ dexamethasone (DEX), and $50 \mu \mathrm{g} / \mathrm{mL}$ ascorbic acid (AA), all from Sigma). The osteogenesis of MC3T3-E1 cells were characterized using quantitative real time polymerase chain reaction (qRT-PCR) and immunofluorescence staining. After culturing the MC3T3-E1 cells on the coating surface for 7, 14 and 21 days the bone associated genes including alkaline phosphatase (ALP), collagen type 1 (Col I), osteopontin (OPN), and osteocalcin (OCN) were confirmed by qRT-PCR. Moreover, the protein expression of OPN at 14 days was also confirmed using fluorescence microscopy.

\subsubsection{Quantitative Real-time PCR Analysis}

The osteogenic differentiation of MC3T3-E1 cells was first analyzed by qRT-PCR. The MC3T3-E1 cells $\left(1 \times 10^{4}\right.$ cell $\left./ \mathrm{cm}^{2}\right)$ cultured on the Chi and MBGN-0.50 coatings for 7,14 and 21 days were collected for the evaluation of osteogenic related gene expression. Briefly, the first strand cDNA was synthesized from 1 $\mu \mathrm{g}$ of RNA using a SuperScript first strand synthesis system for RT-PCR (Bioneer) according to the manufacturer's instruction with a total reaction solution volume of $50 \mu \mathrm{L}$. The qRT-PCR was conducted using SYBER Green qPCR SuperMix reagents (Invitrogen) using 50 ng of total cDNA. Further, relative transcript quantities were calculated using the $\Delta \Delta \mathrm{Ct}$ method, with glyceraldehyde 3-phosphate dehydrogenase (GAPDH) as the endogenous reference amplified from the samples. Fold change of 
corresponding gene was subsequently calculated from $2^{-\Delta \Delta C t}$. The primer sequences specific for the targeted gene including alkaline phosphatase (ALP), collagen type 1 (Col I), osteopontin (OPN), and osteocalcin (OCN) and glyceraldehyde 3-phosphate dehydrogenase (GAPDH) used for qRT-PCR are listed in supplementary Table S1.

\subsubsection{Immunofluorescence Staining}

To confirm the protein expression, immunofluorescence staining was performed at 14 days. For this, MC3T3-E1 cells were culture on Chi and MBGN-0.50 coating surface for 14 days at $37.8{ }^{\circ} \mathrm{C}$ in a humidified $5 \% \mathrm{CO}_{2}$ atmosphere. The samples were permeated with $0.1 \%$ Triton-X 100 in phosphate buffer for $5 \mathrm{~min}$ at room temperature. After washing with PBS for three times being blocked using $1 \%$ BSA in PBS for 30 min. The samples were then incubated in primary antibody (OPN; sc-20788, 1:300) for $1 \mathrm{~h}$ under ambient temperature. Then samples were washed twice with PBS and stained with a fluorescein isothiocyanate (FITC)-labeled secondary antibody for $1 \mathrm{~h}$. Finally, cells were co-stained with phalloidin and cell nuclei with 4',6-diamino-2-phenylindole (DAPI). The fluorescent images were captured by fluorescence microscopy (Olympus DP72, Japan).

\subsection{Statistical Analysis}

All the data are presented as the mean \pm standard deviation. Statistical analysis was carried out using one-way analysis of variance (ANOVA) test and a significance level was considered at ${ }^{*} p<0.05$.

\section{RESULTS}

\subsection{Characterization of MBGN}

The FE-SEM image of MBGN showed a spherical shape with uniform size (Figure 2 a). The HR-TEM morphology of sol-gel derived mesoporous bioactive glass nanoparticles is shown in Figure 2 (b). The TEM results confirmed that the MBGN were monodisperse, with homogeneous spherical shape and uniform size distribution. The size of the nanoparticles analyzed by TEM images (diameters determined was obtained from 50 counts) gave an average size of $90 \pm 10 \mathrm{~nm}$. We also observed pores of few nanometer $(\sim 3-4 \mathrm{~nm})$ in the TEM image. 
The FT-IR spectra of synthesized MBGN and amine surface functionalized mesoporous bioactive glass nanoparticles (MBGN-NH 2 ) are shown in Figure 2 (c). The peaks at $471 \mathrm{~cm}^{-1}$ (Si-O-Si bending), $800 \mathrm{~cm}^{-1}$ (Si-O vibration) and $1095 \mathrm{~cm}^{-1}$ (Si-O-Si stretching), and additional band at $1200 \mathrm{~cm}^{-1}$ and $1640 \mathrm{~cm}^{-1}$ are associated to $\mathrm{N}-\mathrm{H}$ stretching mode. The amination of MBGN was carried out using APTES to allow the cathodic deposition during electrodeposition process. Further, the $\zeta$-potential of the MBGN was measured in distilled water at $\mathrm{pH} 7.4$ changed from highly negative $(-20.2 \mathrm{mV})$ to positive $(+27.4 \mathrm{mV})$ after the amination, and confirmed the successful amine-functionalization of the MBGN (Figure S1). The FTIR and $\zeta$-potential results together confirmed the successful surface modification (amination) of MBGN.

An amorphous silica phase with a broad peak at $2 \theta=22.3^{\circ}$ was observed in the XRD pattern (Figure 2d). Further, the pore size of the MBGN was determined by small angle $\left(1^{\circ}<2 \theta<7^{\circ}\right) X$-ray diffraction (SA$\mathrm{XRD}$ ) analysis. The SA-XRD analysis showed diffraction peaks representative of porous structure (Figure 2e). The SA-XRD analysis, showing a broad peak at $2 \theta=2.82^{\circ}$ for $M B G N$ and $3.24^{\circ}$ for $M B G N-\mathrm{NH}_{2}$, which correspond to d-spacing of 3.13 and $3.19 \mathrm{~nm}$, respectively. Moreover, the reduction of peak intensities of the MBGN-NH 2 confirmed the functionalization of MBGN surface and pores with amine groups ${ }^{28}$.

The $\mathrm{N}_{2}$ adsorption/desorption isotherm curves confirmed the mesoporous structure and reduction of surface area (Figure 2f) and pore size (Figure S2) after amination of MBGN. The isotherm curve of MBGN and MBGN-NH 2 nanoparticles were identical to the curve representing a type IV mesoporous structure. The surface area of the MBGN and MBGN-NH 2 are 375.037 and $347.372 \mathrm{~m}^{2} / \mathrm{g}$, and the pore volume 0.462 and $0.376 \mathrm{~cm}^{3} / \mathrm{g}$ respectively. The Barrett-Joyner-Halenda (BJH) method produced broader pore size distribution of $3.955 \mathrm{~nm}$ and low intense peak at $4.619 \mathrm{~nm}$ in the MBGN (Figure S2). After amination of MBGN, the pore size distribution was narrow and bit sharper at $3.661 \mathrm{~nm}$ and a low intense peak at $2.929 \mathrm{~nm}$ in the MBGN-NH . Overall, the surface area, pore volume, and pore size reduction was confirmed by the BJH and BET results of the corresponding nanoparticles. 
(a)

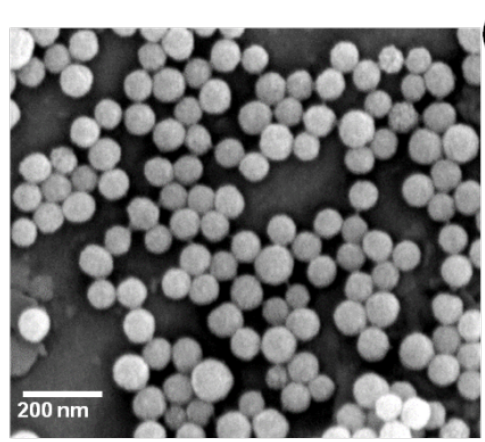

(b)

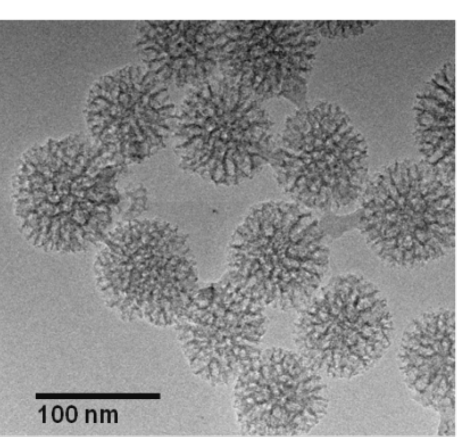

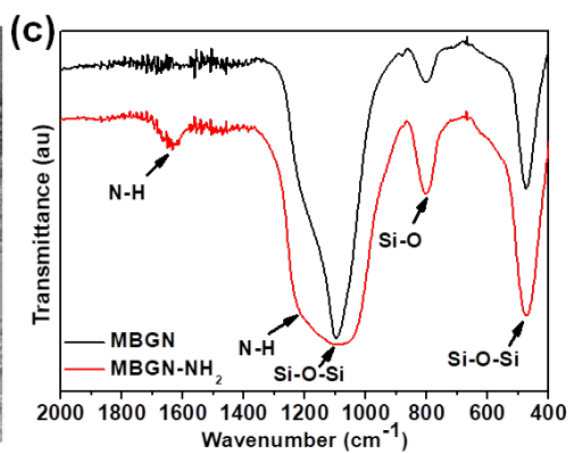

(d)



(e)

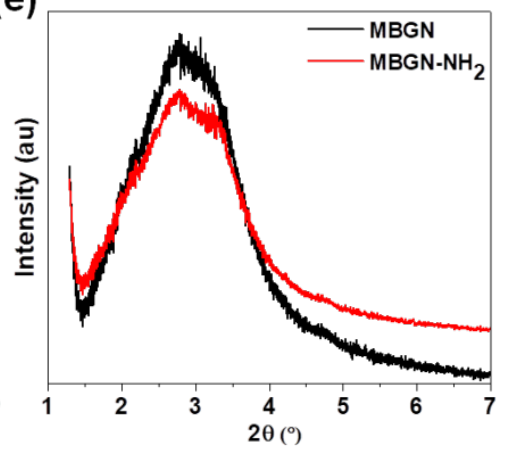

(f)

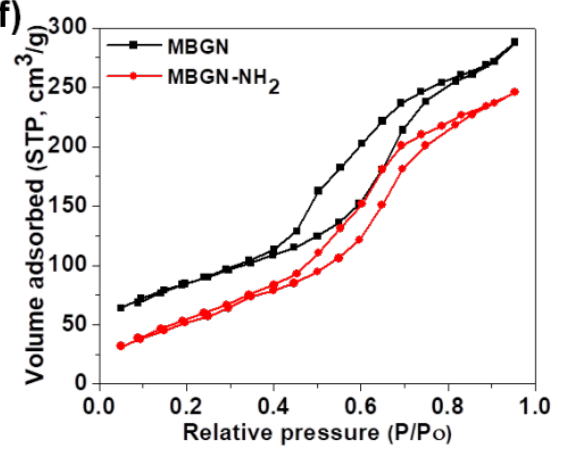

Figure 2. Characterizations of highly mesoporous bioactive glass nanoparticles; (a) SEM and (b) TEM image of MBGN with highly mesoporous structure. (c) FT-IR spectra and (d) XRD patterns, showing amorphous nature of sol-gel synthesized mesoporous bioglass nanoparticles. (e) SA-XRD analysis, showing a broad peak at $2 \theta=2.82^{\circ}$ for $\mathrm{MBGN}$ and $2.76^{\circ}$ for $\mathrm{MBGN}-\mathrm{NH}_{2}$, which correspond to d-spacing of 3.13 and $3.94 \mathrm{~nm}$, respectively. (f) $\mathrm{N}_{2}$ adsorption/desorption isothermal curves.

\subsection{Characterization of MBGN/Chi Coating}

The surface morphology of chitosan and MBGN-Chi composites coatings on titanium were observed by SEM (Figure 3a). Chitosan was uniformly deposited onto the titanium substrate via electrophoretic deposition (EPD) at room temperature. During the EPD process, micro-bubbles were observed at higher applied voltages over 40 volts. This phenomenon is mainly due to hydrogen evolution by hydrolysis of water at cathode and subsequent entrapment of bubbles in the coatings ${ }^{29}$. To overcome this problem, ethanol was used in suspension preparations. A mixture of water-ethanol prevented the bubble formation during electrodeposition. Moreover, the MBG nanoparticles in chitosan solution were kept under sonication in the ice water bath to ensure homogeneous suspension. Ice-water was used to maintain the low temperature to avoid any adverse effect on suspensions due to the generation of temperature by the sonicator. A series of different concentrations of MBGN-Chi suspensions were used for coatings. After 
successful deposition of Chi and MBGN-Chi nanocomposites, we choose, 1:1 concentration ratio (Chi to MBGN; w/w) for the further detailed characterization. The coatings showed mechanically good adherence to the titanium surface. For the best coating, parameters were optimized by trial-and-error method, and the best conditions were $25 \mathrm{~V}$ for $3 \mathrm{~min}$ and maintaining a $10 \mathrm{~mm}$ electrode separation at $3.5 \mathrm{pH}$ of the suspension.

Further, nanotopographical composite coating of MBGN-Chi was examined by X-ray diffraction and ATRFTIR spectrometer. An amorphous peak was observed for MBGN-Chi composite coatings at $2 \theta \sim 21^{\circ}-23^{\circ}$, mainly due to presence of mesoporous bioglass nanoparticles (Figure 3b). ATR-FTIR spectroscopy was used to determine the chemical functional groups bonding in the composite coating. Typical FTIR spectra of chitosan film and MBGN-Chi composite coatings on the titanium substrate are shown in Figure 3c. The peaks around $894 \mathrm{~cm}^{-1}$ and $1155 \mathrm{~cm}^{-1}$ corresponded to the saccharide structure, mainly due to the vibrations of the glycosidic bonding (-C-O-C-) which occurs between the repeating unit from chitosan. The broad peaks at $1015 \mathrm{~cm}^{-1}$ and $1075 \mathrm{~cm}^{-1}$ are due to the C-O stretching vibration in chitosan ${ }^{30}$, at 1640 $\mathrm{cm}^{-1}$ and $1580 \mathrm{~cm}^{-1}$ correspond to amide I and amide II, respectively ${ }^{31}$. However, most of the peaks from the MBGN-Chi composite coatings either disappear or have very low intensity. A broad peak at $1053 \mathrm{~cm}^{-1}$ and a small at $785 \mathrm{~cm}^{-1}$ are due to Si-O-Si stretching and vibration, respectively, and a sharp peak at 445 $\mathrm{cm}^{-1}$ is due to Si-O-Si bending ${ }^{32}$. The TG analysis of the various coatings is shown in the Figure $3 \mathbf{d}$. The thermal degradation of $\mathrm{Chi}$, MBGN-0.25, MBGN-0.50, and MBGN-0.75 coatings underwent weight loss in three stages: first stage showing an initial $18.69 \%, 10.1 \%, 25.4 \%$ and $9.8 \%$ weight loss between 25 to $150{ }^{\circ} \mathrm{C}$ for Chi, MBGN-0.25, MBGN-0.50, and MBGN-0.75, respectively, which represent the evaporation of physisorbed water molecules; second stage $20.4 \%, 48.73 \%, 12.74 \%$, and $20.8 \%$ weight loss between 150 to $350{ }^{\circ} \mathrm{C}$, and third stage between 350 to $600{ }^{\circ} \mathrm{C}$ which represent thermal degradation of chitosan; which correspond to the carbonization of residual organic components. At $600{ }^{\circ} \mathrm{C}$, Chi showed $7.69 \%$ and all other composites also preserved certain amount of weight which corresponds to the remaining weight of the MBGN in the coatings. The remaining weight was $28.49 \%, 39.34 \%$, and $56.96 \%$ for MBGN-0.25, MBGN-0.50\%, and MBGN-0.75\%, respectively. These remaining amounts of the weight indicate that the MBGN was homogenously present in the coatings and preserved the initial compositional ratios. 

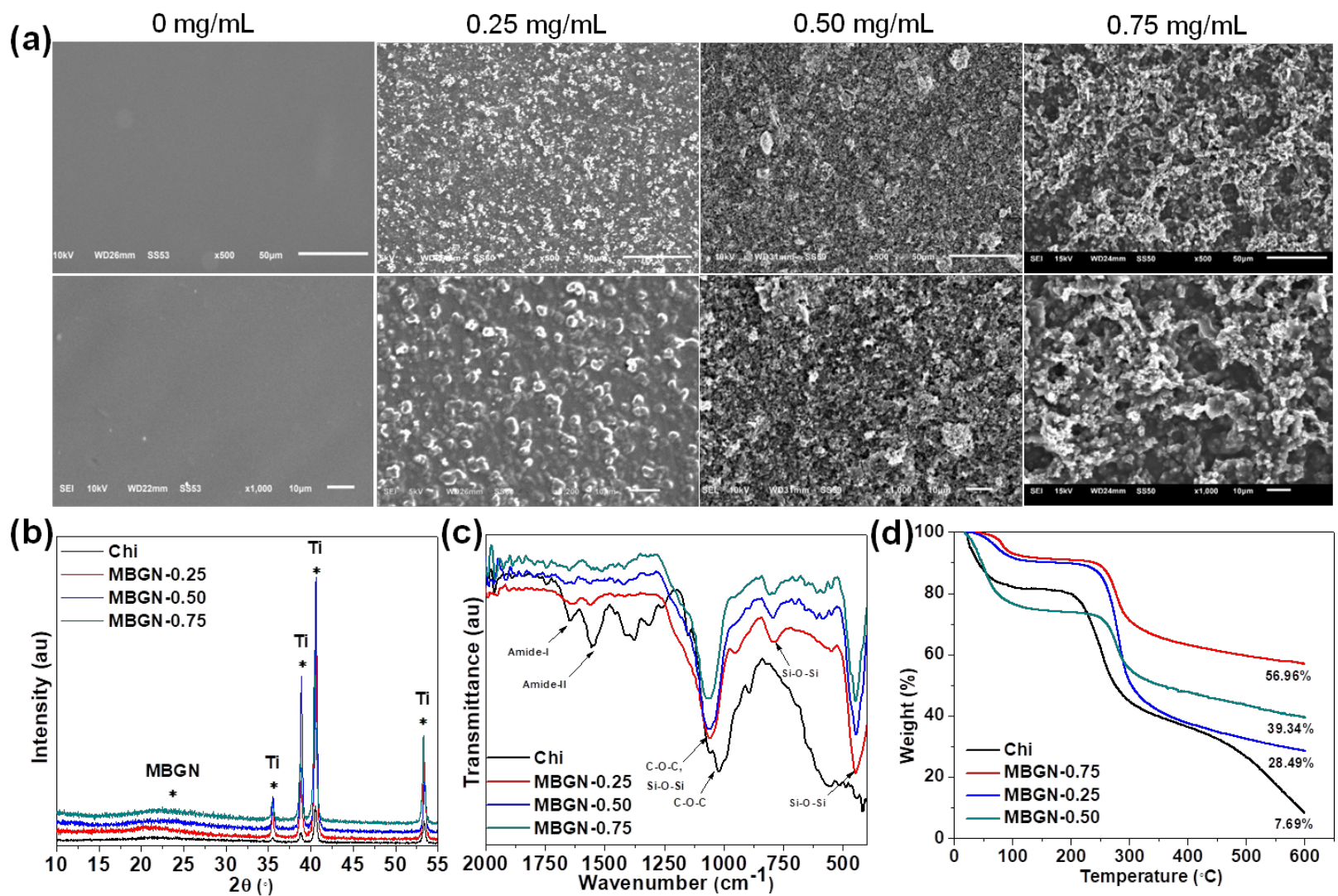

Figure 3. Characterization of nanotopographical composite coatings; (a) SEM images of Chi and MBGNChi composite coatings surface at low and high magnifications. A 1:1 ratio of MBGN and Chi i.e. 0.5 $\mathrm{mg} / \mathrm{mL}$ concentration, ratio was chosen after depositing of various concentrations and measuring their stability and surface morphologies. (b) XRD patterns of chitosan and MBGN-Chi coatings on titanium substrate. (c) ATR-FTIR spectra of chitosan and MBGN-Chi coatings. (d) TG analysis of chitosan and MBGN coatings.

We further evaluated the kinetics of EPD by measuring the weight gain during the electro-coating process. The weight gain was measured by varying the deposition time and applied voltage across the electrode at a constant $\mathrm{pH} 3.5$ of EPD suspension. For this, EPD suspension of Chi $(0.5 \mathrm{mg} / \mathrm{mL})$ and MBGN-Chi $(1: 1 ; 0.5 \mathrm{mg} / \mathrm{mL})$ solution was prepared and maintained at $\mathrm{pH} 3.5$. A slight change in $\mathrm{pH}(0.1$ $0.2)$ was observed before and after the EPD process. At a constant applied voltage ( $25 \mathrm{~V})$, the weight of the coating for Chi and MBGN-Chi increased with increasing deposition time from 1 to 5 min (Figure 4a). The weight gain was more pronounced in the MBGN-Chi composite coating as the positively charged MBGN and protonated Chi molecules move towards the cathode and deposit with increasing deposition time. A linear coating weight gain was also observed as a function of time and voltage. An observation of the weight gains at constant deposition time (at $5 \mathrm{~min}$ ) with varying applied voltage revealed that the 
coating weight gain is linear until 25 volts and above become saturated (Figure 4b). The MBGN addition increases the weight of composite coating, which can be explained based on coating thickness as well as deposition longer time and higher applied voltage.

(a)

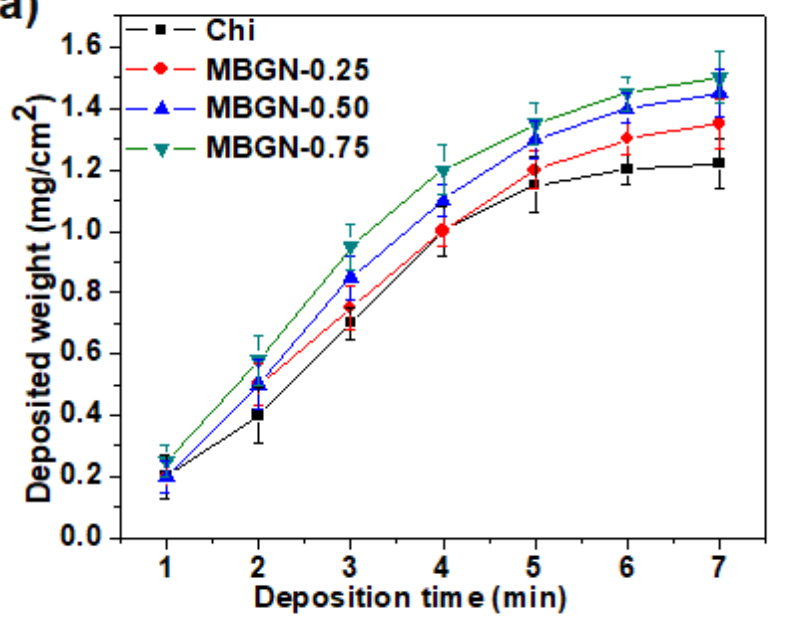

(b)

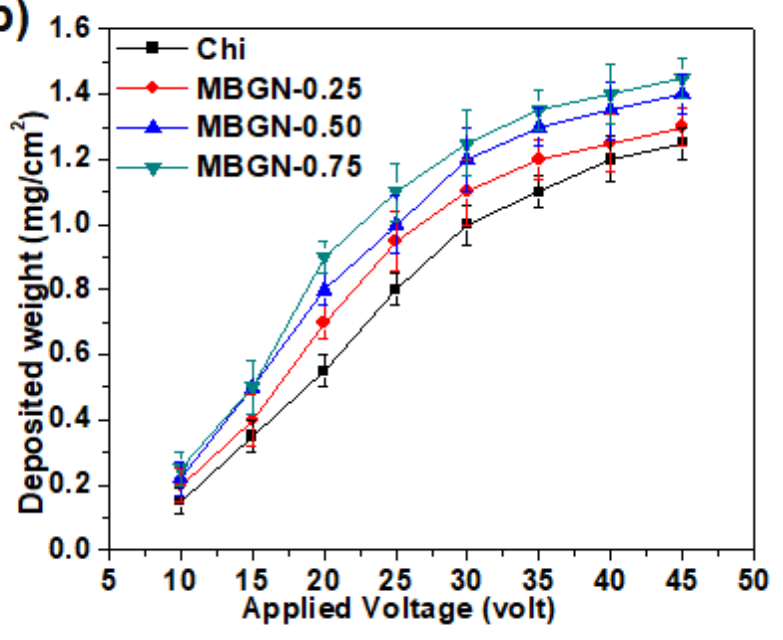

Figure 4. Weight gains during the EPD process for varying deposition time and applied voltage for Chi and MBGN-Chi composite coatings; (a) deposited weight for varying time and constant voltage at $25 \mathrm{~V}$ and $\mathrm{pH} 3.5$, and (b) deposited weight for varying applied voltage and at constant deposition time for 5 min and $\mathrm{pH} 3.5$.

\subsection{Mechanical Strength and Nanoroughness}

The mechanical effects of the MBGN in the MBGN-Chi coating were then investigated by nanoindentation. The loading-unloading curves from loading with Berkovich indenter into Chi and MBGN-0.25, MBGN-0.50, and MBGN-0.75 coatings up to $3000 \mu \mathrm{N}$ were carried out (Figure 5a). The plasticity of the different coatings is calculated from the final depth. The chitosan coating showed small plastic deformation with a final depth of $575.6 \mathrm{~nm}$. MBGN-0.25, MBGN-0.50, and MBGN-0.75 coatings showed greater plastic deformation with final indentation depths of $1010.14,1624.71$, and $2189.72 \mathrm{~nm}$, respectively. The loading-unloading (load vs. depth) results of composite coatings demonstrated the significant role of MBGN and confirmed the higher MBGN concentration coatings have higher indentation depth.

The hardness vs. indentation depth of the various coatings results showed the material becoming softer as the MBGN content increased (Figure 5b). MBGN-0.75 is the softest coatings, with a hardness of $0.0309 \pm 0.016 \mathrm{GPa}$, closely followed by MBGN-0.5 and MBGN-0.25, with a hardness of $0.0408 \pm 0.0121$ and $0.04455 \pm 0.0131 \mathrm{GPa}$, respectively. The chitosan coating exhibited highest hardness of $0.19 \pm 0.1$ 
GPa. Further, the elastic modulus vs. indentation depth of the corresponding coatings was also analyzed (Figure 5c). Chitosan coating exhibits the highest elastic modulus of $5.883 \pm 0.125 \mathrm{GPa}$, and MBGN-0.25, MBGN-0.50, and MBGN-0.75 are $1.085 \pm 0.223,1.125 \pm 0.481$, and $1.19 \pm 0.516 \mathrm{GPa}$, respectively. The scanning probe microscopy (SPM; Hysitron, USA) images of the indenter mark (before and after with 2D and $3 \mathrm{D}$ profile) for Chi and MBGN-0.5 coatings are obtained at load $3 \mathrm{mN}$ (Figure $5 \mathrm{~d}-\mathbf{e}$ ) and the corresponding indentation profile (Figure $\mathbf{5 f - g}$ ). The nanoroughness for the various coatings is listed in Table 1. The average roughness $(\mathrm{Ra})$ for Chi coating was lowest $(8.6070 \pm 2.7825 \mathrm{~nm})$ and MBGN-0.50 maximum (23.1785 $\pm 3.7263 \mathrm{~nm})$. The average nanoroughness for MBGN-0.25 and MBGN-0.75 coating were $10.8352 \pm 2.3759 \mathrm{~nm}$ and $13.3995 \pm 3.8424 \mathrm{~nm}$, respectively.
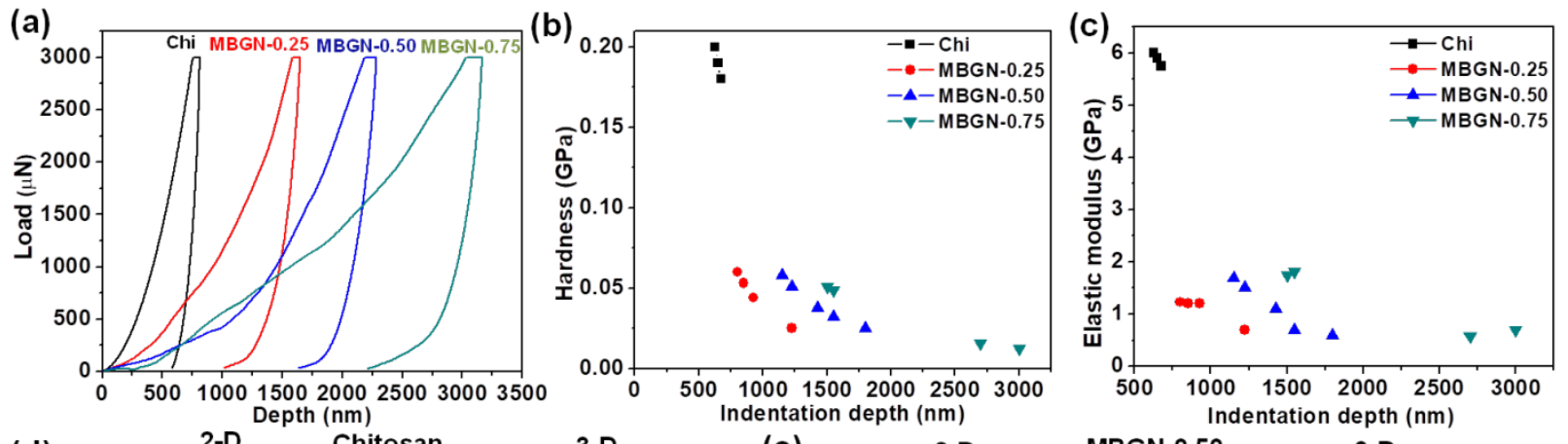

(d)

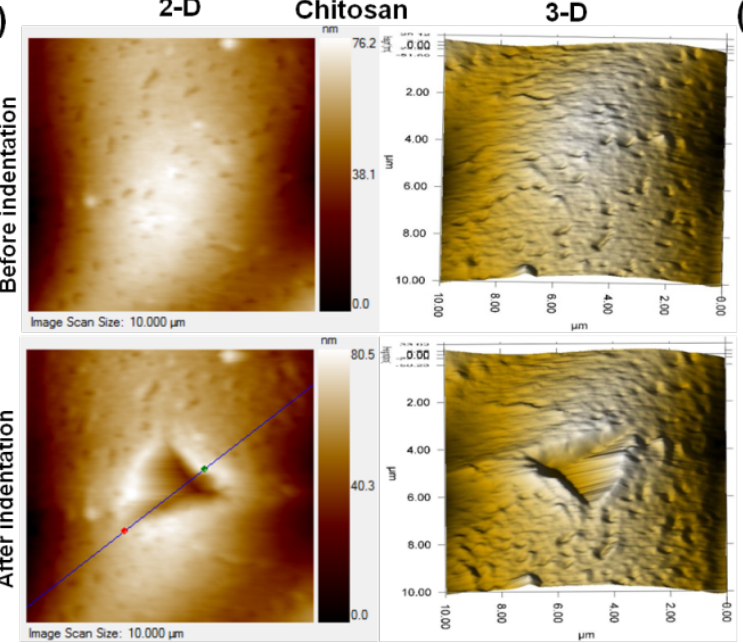

(f)

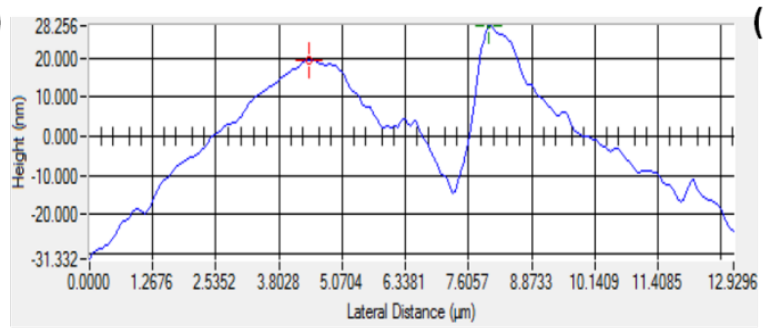

(e) 2-D
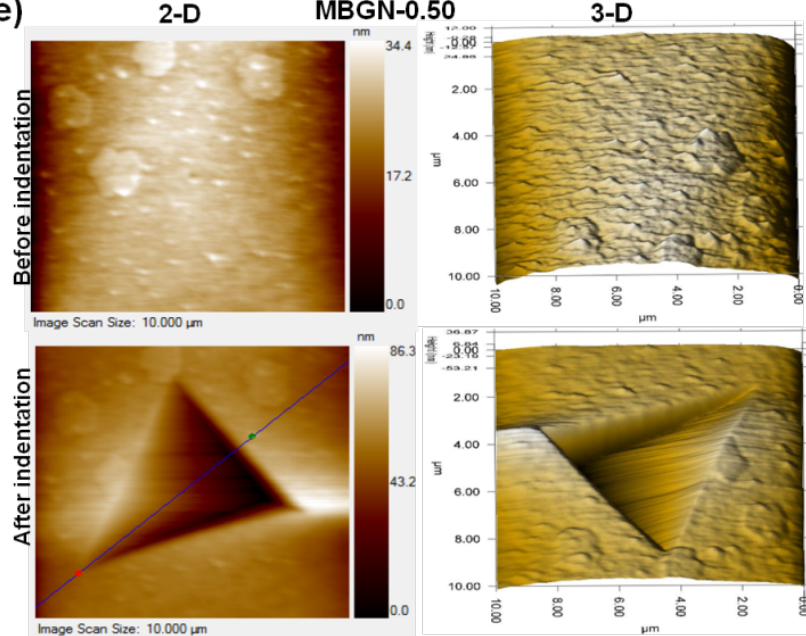

(g)

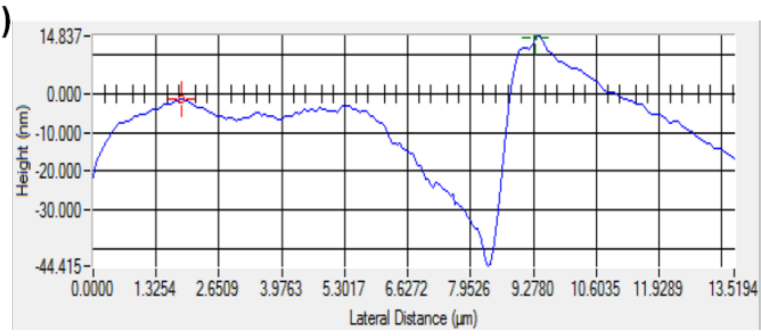


Figure 5. Mechanical strength and nanoroughness; (a) Load-depth curve for Chi and varying MBGN (0.25-0.75) composite coatings, (b) Nano-hardness and (c) elastic modulus, (d-e) surface topography of chitosan and MBGN-0.50 coatings with $2 \mathrm{D}$ and $3 \mathrm{D}$ view before and after indentation; nanoindentation profile of indents for (f) Chi, and (g) MBGN-0.50 at $3 \mathrm{mN}$ peak load.

Table 1 Nanoroughness of the coatings measured by nanoindentation.

\begin{tabular}{lll}
\hline Sample & RMS roughness $(\mathbf{R q})(\mathbf{n m})$ & Average roughness $(\mathbf{R a})(\mathbf{n m})$ \\
\hline Chi & $12.4581 \pm 3.0145$ & $8.6070 \pm 2.7825$ \\
MBGN-0.25 & $12.8651 \pm 2.4381$ & $10.8352 \pm 2.3759$ \\
MBGN-0.50 & $32.6728 \pm 4.8317$ & $23.1785 \pm 3.7263$ \\
MBGN-0.75 & $15.2739 \pm 4.3782$ & $13.3995 \pm 3.8424$ \\
\hline
\end{tabular}

\subsection{Bone-bioactivity in SBF}

The apatite forming ability of the nanotopological composite coatings surface was assessed in $1 \mathrm{X}$ SBF solution. The SEM images (low and high magnification) of the coating showed the MBGN-Chi samples were covered with apatite after immersion in SBF solution for 3 and 7 day (Figure 6a). Previously, it was reported that bioglass composite coatings have apatite-forming ability with better cell proliferation and differential potential in vitro ${ }^{32}$. Therefore, it is reasonable to expect that the microporous coating surface may have satisfactory performance in vitro. Furthermore, a higher magnification SEM images revealed the mineral phase and structure of nanocrystallites, as has been typically observed in biomimetically mineralized apatite and also confirmed the mineral was spherical and not an agglomeration of nanocrystals. Moreover, the mineralized coating phases were analyzed by XRD and ATR-FTIR. The XRD results show two peaks at $2 \theta=26.3^{\circ}$ and $32.4^{\circ}$, which are characteristic peaks of apatite ${ }^{33}$. These peaks with increasing immersion time became sharper and intense and more apparent (Figure 6b), suggesting strong apatite deposition under physiological conditions in SBF solution. This rapid apatite formation on the nanotopological composite coatings can be explained on the basis of ion ( $\mathrm{Ca}, \mathrm{Si})$ release mechanism from the MBGN. ATR-FTIR spectra also revealed bands related to apatite $\left(600,959\right.$, and $1014 \mathrm{~cm}^{-1}$ correspond to $\mathrm{v}_{2} \mathrm{P}-\mathrm{O}$ bending and $\mathrm{v}_{1} \mathrm{P}-\mathrm{O}$ and $\mathrm{v}_{3} \mathrm{P}-\mathrm{O}$ stretching, respectively) after the immersion, and the band intensities also increased with time (Figure 6c). Moreover, the bands at 870 and $1410 \mathrm{~cm}^{-1}$ 
were assigned to $\mathrm{v}_{2} \mathrm{C}-\mathrm{O}$ and $\mathrm{v}_{3} \mathrm{C}-\mathrm{O}$ stretching vibration mode of $\mathrm{CO}_{3}{ }^{2-}$, signifying the incorporation of a carbonate group in the apatite crystal lattice ${ }^{9,25-26}$.
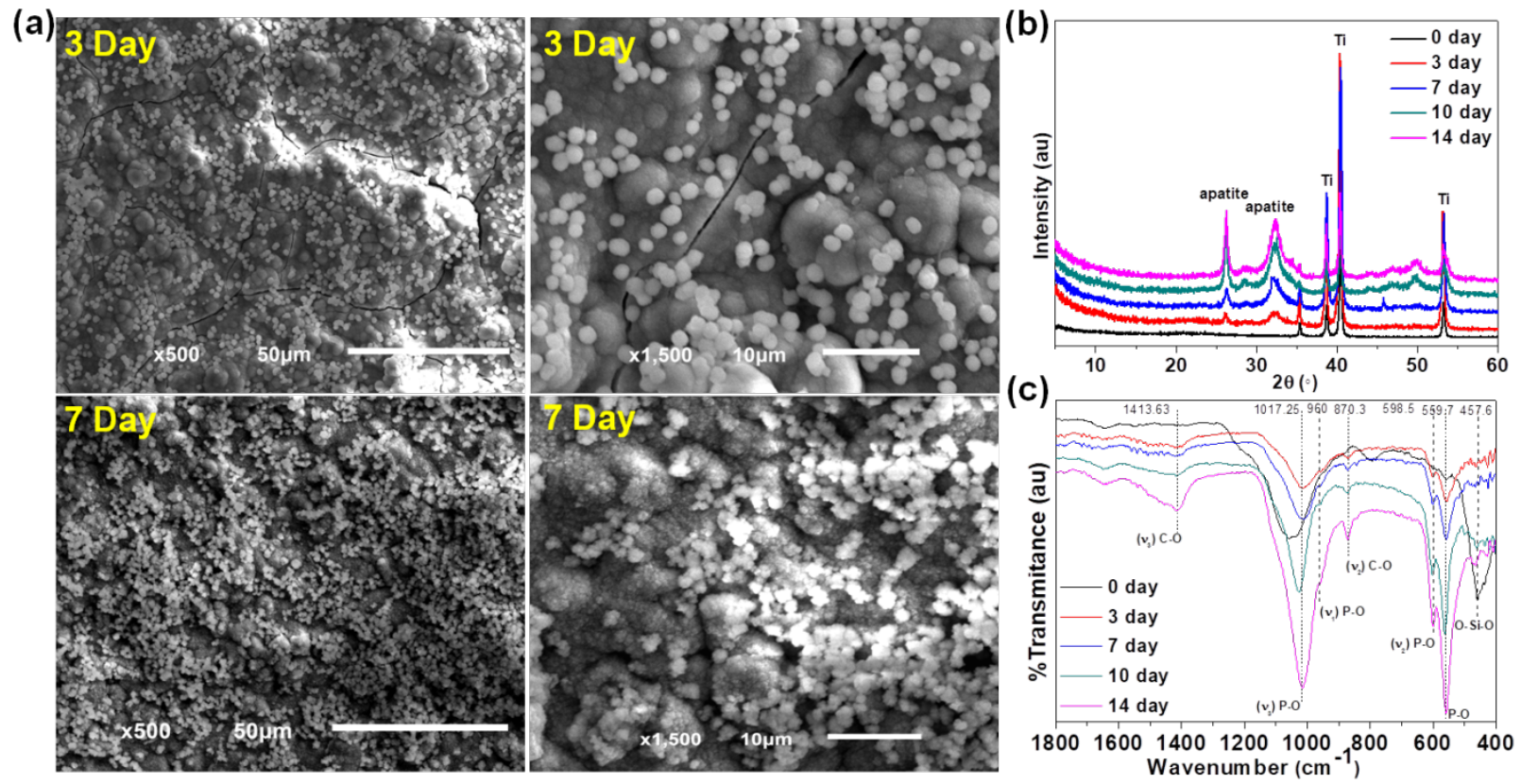

Figure 6. Apatite-forming ability of MBGN-0.50 nanocomposite coatings; (a) SEM images of MBGN-0.50 composite coatings after immersion for 3 and 7 days in $1 \mathrm{X}-\mathrm{SBF}$ solution at $37^{\circ} \mathrm{C}$ with low and high magnifications showing apatite crystal formation over the coating surface. (b) XRD patterns of MBGN0.50 composite coatings after emersion in SBF at different time points showed apatite related peaks. (c) ATR-FT-IR spectra of mineralized coating surface also confirmed the same.

\subsection{Cell Adhesion, Spreading and Proliferation}

The initial cell adhesion and spreading were assessed by staining with phalloidin and DAPI to visualize the cytoskeleton and nuclei, respectively, and the results are shown in Figure 7. Schematic representation showed that the cell behavior on Chi (smooth) surface and MBGN-0.50 (nanotopography) (Figure 7a). The MC3T3-E1 cells on pure Chi deposited sample showed mixed morphology with extended cytoskeleton at $24 \mathrm{~h}$. However, the cells on MBGN-Chi nanotopographical composite coating exhibits mostly spindle shape morphology with extended cytoskeleton and filopodia extensions. The difference in the expression of cytoskeleton of cells is significantly different at $24 \mathrm{~h}$ and Chi surface exhibits polygonal shape with other different morphology and MBGN-Chi surface exhibits spike and spindle shape (Figure 7b). These results indicate that the nanotopography of MBGN-Chi surface is more 
favorable to initial cell adhesion and spreading. These results may be ascribed to the effects of mesoporous bioglass nanoparticles in the chitosan matrix. The relatively more positive zeta potentials of the MBGN-Chi surface is also helpful for initial cell adhesion ${ }^{34}$.

We next address the cell adhesion to the surface with the help of DAPI stained nuclei (nucleus in blue). From the images, we counted the cell number anchored to the Chi and MBGN-Chi samples (Figure $\mathbf{7 b}$ ). There was significantly higher number of cells adhered to the MBGN-Chi nanotopographical composite coatings compare to pure Chi surface after $24 \mathrm{~h}$ of cell culture. Additionally, the cell spreading area was also examined. The cell spreading area was quantified from images (Figure 7c). The cell spreading area for MBGN-Chi surface was significantly lower and spread in spiked in shape. Previously, we have observed that the nanotubular and nanostructure surface topography affect initial cell adhesion and spreading significantly and regulate the cell behavior ${ }^{9,14}$.

(a) Smooth surface

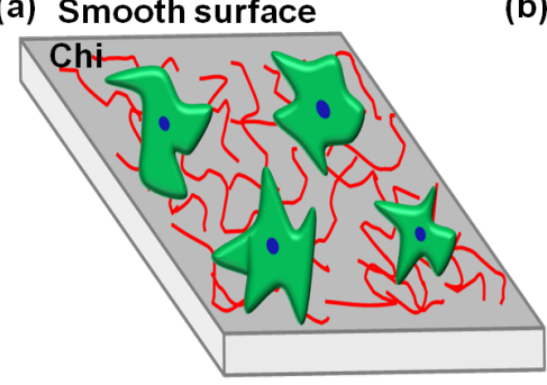

Nanotopography surface

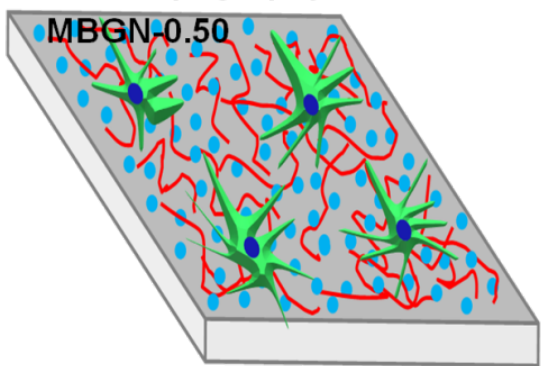

(b)
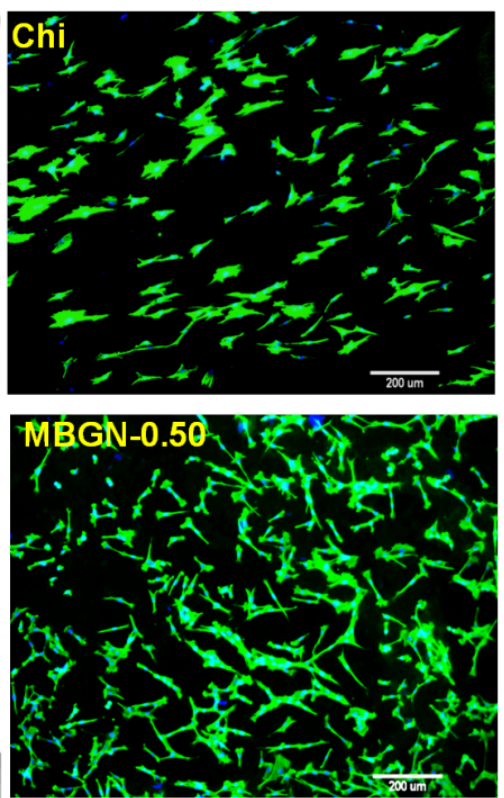

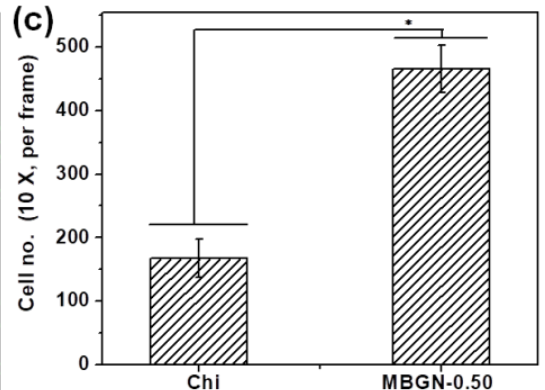

(d)

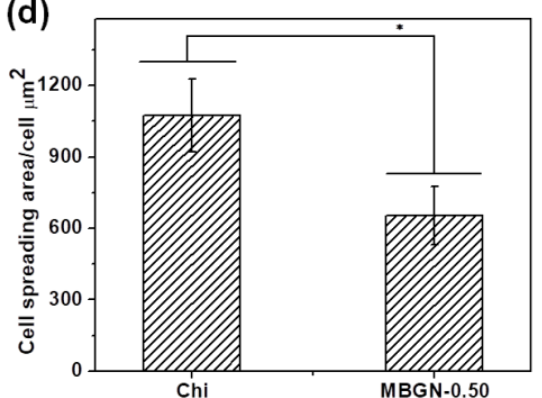

Figure 7. Cell adhesion and spreading morphology at $24 \mathrm{~h}$; (a) Schematic representation of cell behavior on Chi (smooth surface) and MBGN-0.50 (nanotopography surface), (b) fluorescence images of cells stained with phalloidin (green) and the nucleus with DAPI (blue), (c) the cell adhesion numbers, and (d) the cell spreading area quantified. The cell number were counted from DAPI stained images $(n=3,10 X)$ and corresponding cell spreading area was evaluated by use of ImageJ software. Statistical significance difference was noted $\left({ }^{*} p<0.05\right)$.

The cell proliferation rate on the coatings was quantified by means of a CCK assay with culture for up to 7 days (Figure 8a). An increase of the cell numbers level with culture time for both coatings was evident for 
up to 7 days, demonstrating that both coatings provided favorable substrate conditions for the growth of cells with no significant inhibition of cell proliferation. Moreover, MBGN-Chi nanocomposite coatings exhibited statistically significantly higher cell proliferation compared to the Chi coatings. This result indicates that MBGN-Chi coatings are favorable coating surface to the growth of MC3T3-E1 cells without any cytotoxic effect.
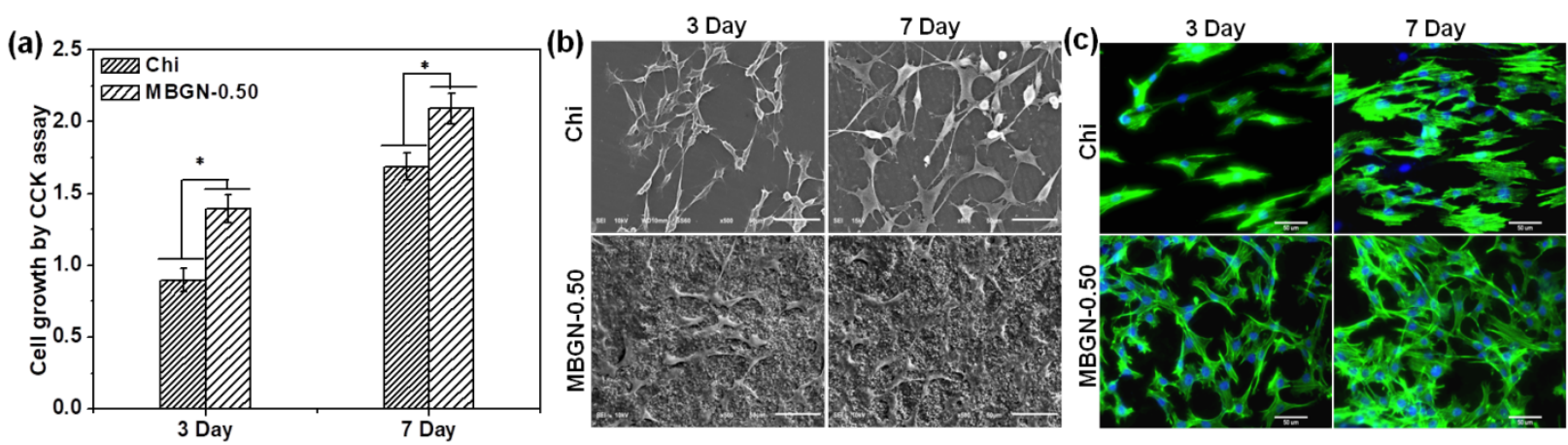

Figure 8. Cell proliferation and morphologies; (a) Cell proliferation using CCK assay for up to 7 days. (b) SEM images of MC3T3-E1 cells cultured on Chi coatings (upper row), and MBGN-0.50 coatings (lower row) after 3 and 7 day of cultivation. (c) Fluorescence images of MC3T3-E1 cells cultured on the coatings (Chi; upper row) and MBGN-0.50 (lower row) for 3 and 7 days; cells adhered and spread onto both coating surfaces. Cells were stained with phalloidin (green) and DAPI (blue) with high magnifications $(40 \mathrm{X})$. Results represented with respect to the chitosan and MBGN-0.50 samples. $\left({ }^{*} P<0.05, \mathrm{n}=3\right.$ ). Scale bars $=50 \mu \mathrm{m}$.

The cell morphology on Chi and MBGN-0.50 coating surfaces after culturing for 3 and 7 days was examined by SEM and fluorescence microscopy, respectively. The SEM images of MC3T3-E1 cells were observed after 3 and 7 days of cell culture on samples of Chi and MBGN-0.50 (Figure 8b). At day 1, cells grew spherical and flat on Chi coatings; while cells were well spread on MBGN-0.50 nanocomposite coatings with long thread-like morphology anchored to spherical bioglass bundles (Figure 7 a-b). At day 3, cell shapes on Chi and MBGN-0.50 composite coatings are more spike and spindle-like in shape. At day 7, cell morphology on Chi coatings are spindle with thin pseudopods and prolonged thread-like morphology, however, cells on nanotopographical MBGN-0.50 composite coatings are dumbbell-like in shape during spreading. These cell morphologies indicate the fully differentiated osteoblasts embedded in the bone matrix ${ }^{35}$. Previously, we have reported that presence of small amount of BGN in coatings significantly improved the in vitro apatite-forming ability and osteogenic differentiation ${ }^{32}$. 
To further understand the impact of nanostructured surface topography on the cells, it was evaluated using fluorescence microscopy (Figure 8c). The cell morphologies on pure Chi coatings exhibited somewhat limited spreading morphology compared to those on MBGN-0.50 composite coatings, which expressed highly extended cytoskeleton processes at day 3, and such cell behaviors were more pronounced with time. At day 7 , cells with many connections completely covered the MBGN-0.50 nanocomposite surface and a similar trend with different cell morphology was observed on Chi surface. These results indicate that the MBGN-0.50 nanostructured coatings promote MC3T3-E1 cells adhesion, proliferation and spreading.

\subsection{Ions Release Profile and Osteogenic Differentiation}

The ICP-AES data shows that $\mathrm{Ca}$ and $\mathrm{Si}$ ions are released from MBGN-0.50 coatings (Figure 9a). The release profiles of calcium ion is rapid up to 14 days and then saturated, while silica ions release rate is rather rapid initially (up to 14 days), and then decreases slightly. This rapid initial release of ions is from the highly presence of MBGN in the coating nanotopological surface and sustained due to controlled degradation of coatings. The resultant calcium and silicate ions release for 21 days is $\sim 242$ and $807 \mathrm{ppm}$, respectively. The Si and $\mathrm{Ca}$ ionic release from MBGN-0.50 coatings has shown stimulatory effects on the proliferation of osteogenic differentiation of MC3T3-E1 cells. Schematic representation showed that the Si and $\mathrm{Ca}$ ions release from substrate $\mathrm{MBGN}-0.50$ (Figure $9 \mathrm{~b}$ ) and its response to stimulate osteogenic differentiation.

(a)

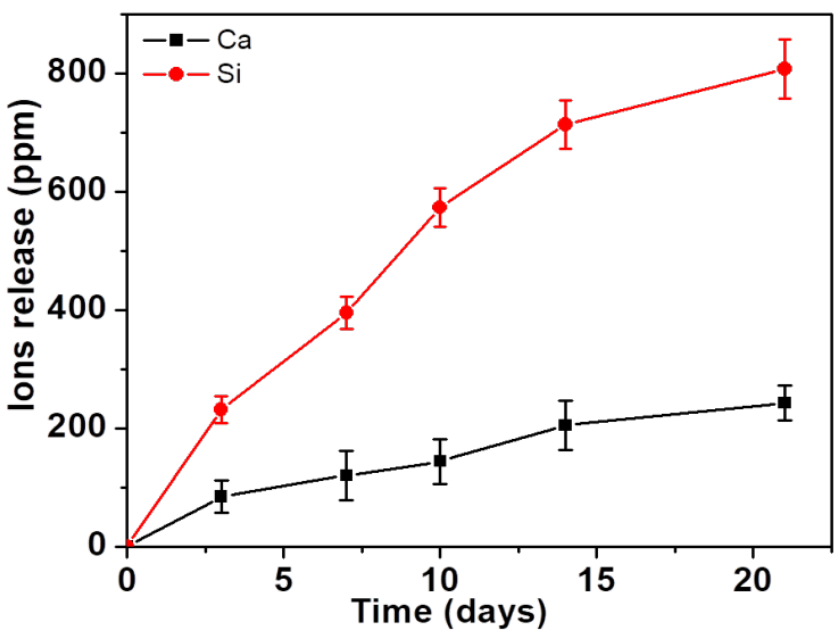

(b)

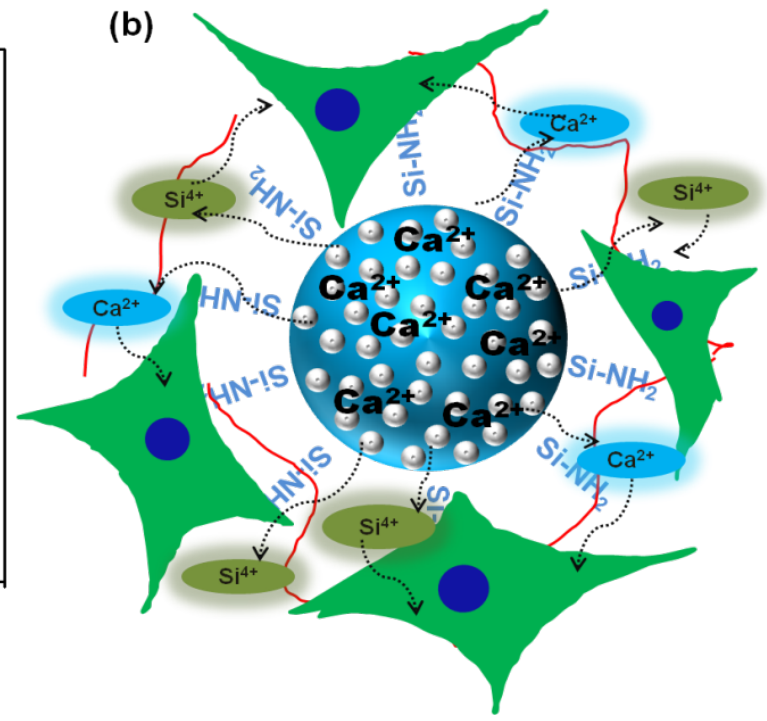


Figure 9. (a) lons (calcium and silicate) release from the coatings up to 21 days. (b) Schematic illustration of the current study on the nanocomposite coatings targeting osteogenic differentiation. The aminefunctionalized MBGN facilitates chemical links with protonated amines of chitosan molecules, which enables physico-chemical stability of the coatings.

Alkaline phosphatase activity (ALP) is an important marker for osteogenic differentiation. At 7 days of cell culture, the cells already produced a significantly high level of ALP on MBGN-0.50 composite coatings compare to pure Chi coatings (Figure 10a). At 14 days, a similar trend was also observed. The MBGN0.50 coated surfaces produce more ALP than Chi coatings, indicating that MBGN-0.50 composite can increase synthesis of ALP. This result confirmed that the presence of MBGN in chitosan matrix significantly enhanced the ALP activity and nanotopographical MBGN-0.50 composite coating played an important role in promoting osteogenic differentiation.

Next, the osteogenic differentiation of MC3T3-E1 cultured on the coatings was further assessed at both gene and protein levels by the q-RT-PCR and immunofluorescence, respectively. The expression of bone associated genes, including ALP, Col I, OPN, and OCN was quantified at days 7, 14 and 21 (Figure 10b). Between Chi and MBGN-0.50, MBGN-0.50 expressed the higher level for all the genes measured at the different time points. Compared to Chi coatings, MBGN-0.50 coatings showed significantly higher expression of ALP, OPN and OCN at every time points. However, Col I showed significantly higher expression at day 14 only. The MBGN-0.50 expressed the highest level for ALP, Col I, genes particularly at day 14; the stimulation was as high as 4.2 and 10.6 fold for ALP and Col I, respectively. Moreover, the highest level for OPN and OCN genes at day 21 and the stimulation was as high as 25 and 17.7 fold, respectively. The secretion of osteogenic protein OPN was confirmed qualitatively by immunocytochemistry at days 14 (Figure 10b). The result of OPN secretion on Chi coating was negligible compared to the MBGN-0.50 coatings. These results suggest that the presence of MBGN in the coatings enable the expression of higher OPN levels. 
(a)

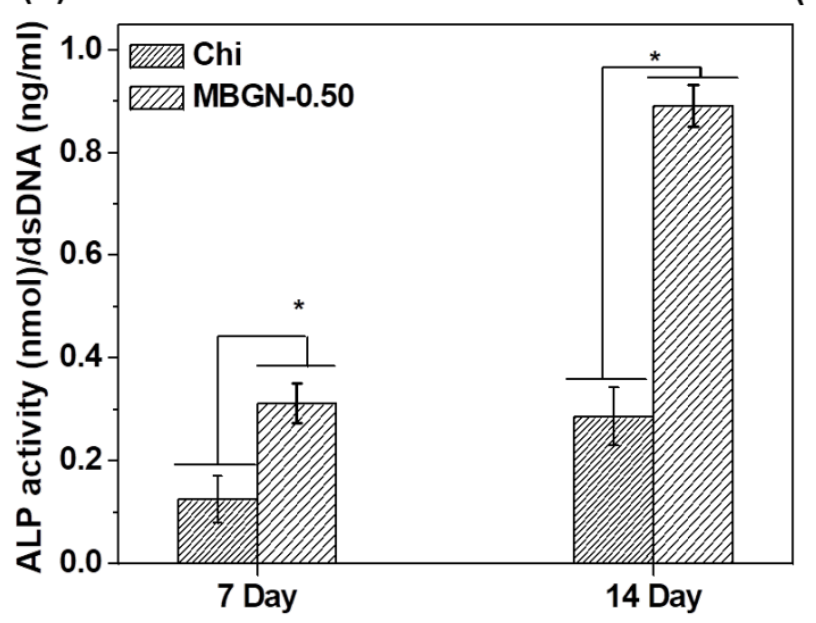

(c)

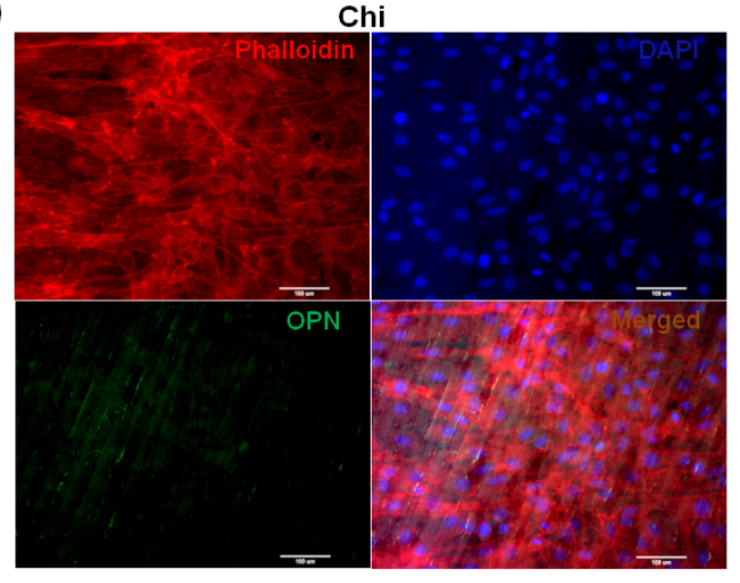

(b)
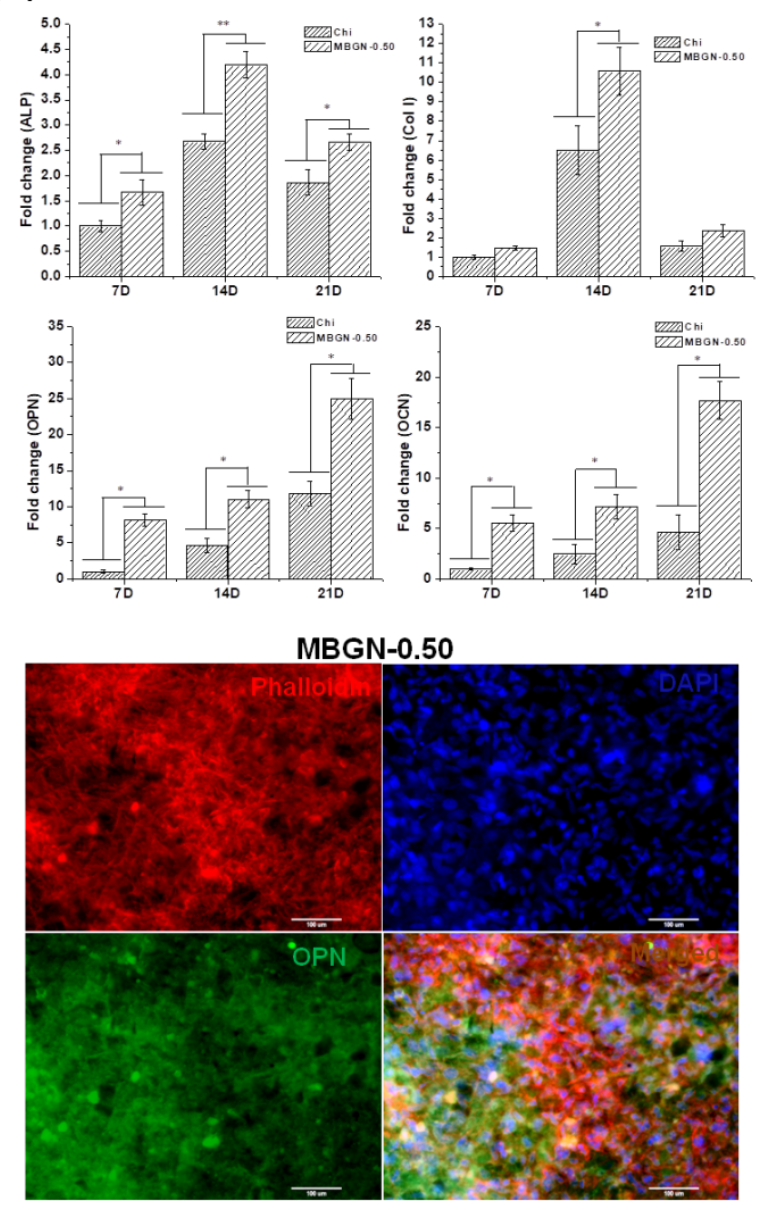

Figure 10. Osteoblastic differentiation of MC3T3-E1 cells, (a) ALP activity of MC3T3-E1 cells assessed on the EPD coatings with $\mathrm{Chi}$ and MBGN-0.50 coatings. Cells were cultured for up to 14 days in the DMEM cell culture and the ALP activity was assessed at 7 and 14 days $\left({ }^{*} P<0.05, n=3\right)$. (b) mRNA levels of the osteogenic genes, including alkaline phosphatase (ALP), collagen type I (Col-I), Osteopontin $(\mathrm{OPN})$ and osteocalcin $(\mathrm{OCN})$, during culture for up to 21 days. ( ${ }^{*} P<0.05, \mathrm{n}=3$ ), (c) Osteopontin immunofluorescence staining assessed on Chi and MBGN-0.50 coatings for 14 days.

\section{DISCUSSIONS}

An important finding of this study is that the MBGN-Chi (particularly MBGN-0.50) coating on titanium showed better osteogenic differentiation compared to pure chitosan coatings. The reason for this enhanced osteogenic differentiation can be understood in terms of the following properties, chemistry of the MBGN-Chi coatings, surface roughness and topography, size (nano-effect) and stiffness of the coatings. Traditional bioglass is similar in composition to the MBGN; however, MBGN exhibits superior bioactivity owing to its large surface area, pore volume and porous structure. We also modified the MBGN 
surface with amine group $\left(-\mathrm{NH}_{2}\right)$ because $-\mathrm{NH}_{2}$ has been shown to enhance osteogenic differentiation of human mesenchymal stem cells ${ }^{36-37}$. Moreover, $-\mathrm{NH}_{2}$ group also has the strongest capability to adsorb fibronectin (FN) when compared to other chemical groups and plays a critical role in integrin binding cell adhesion, proliferation and differentiation ${ }^{38}$. Altering the MBGN surface charge from negative to positive helps in better interaction with negative charged cell membrane. Hence, the intrinsic properties of aminated MBGN have the ability to enhance both the MC3T3-E1 cell adhesion and its corresponding osteogenic differentiation.

The coating of the MBGN with chitosan, XRD and ATR-FTIR were performed to confirm the phase and functional groups of the coatings. The presence of the bioglass nanoparticles in coatings alters the surface topography and consequently the roughness of the coatings. The mechanical stability and nanoroughness of the coatings was analyzed by nanoindentation. The mechanical results suggest as the MBGN concentration increases the hardness and the elastic modulus of the composite coatings decreases. For imposed load $3 \mathrm{mN}$, triangular indents are clearly observed with depths varying with respect to MBGN concentrations. No cracks were observed around the indented area for Chi, MBGN0.25 and MBGN-0.50 coatings. However, we observed no clear triangular indent in case of MBGN-0.75 coatings (Figure S3). Moreover, the SPM images showed that the coating was pile-up at $3 \mathrm{mN}$ indentation load. The obtained hardness of the coatings agrees well and falls within the reported range ${ }^{39-40}$. However, the elastic modulus of the coatings is lower than reported values ${ }^{41}$. A $2 D$ and $3 D$ view of the samples before and after the indentation experiment showed the homogeneous coating and uniform distribution of the MBGN. The 2D views (before and after indentation) of the coatings showed homogeneous and uniform coating without any cracks. The top 3D view of the coatings (before and after indentation) showed smaller homogeneous distribution of the MBGN in MBGN-0.5 coatings, indicating the importance of MBGN in the coatings as nanoroughness. The nanoindentation profile of the Chi coatings showed less height compare to the MBGN-0.50 coating. The higher triangle size in the MBGN-0.50 coatings suggest the coatings are softer compared to Chi coating. This can be explained on the basis of inclusion of MBGN in coatings which generated nanotopography and highly rough surface compared to Chi alone. The subsequent nanotopography and nanoporous structure reduced the hardness of the coatings. The SPM image of MBGN- 0.75 coating showed a completely ruptured surface and no obvious 
triangle can be observed, indicating a weak coatings surface. The nanoroughness for the various coatings are listed in Table 1. The average roughness $(\mathrm{Ra})$ for Chi coating was lowest $(8.6070 \pm 2.7825$ $\mathrm{nm})$ and MBGN-0.50 maximum (23.1785 $\pm 3.7263 \mathrm{~nm})$. The average nanoroughness for MBGN-0.25 and MBGN-0.75 coatings were $10.8352 \pm 2.3759 \mathrm{~nm}$ and $13.3995 \pm 3.8424 \mathrm{~nm}$, respectively. These results regarding average nanoroughness indicates that up to a limit of MBGN concentration roughness increases but when MBGN content is higher than chitosan, coating become weak and subsequently roughness decreased abruptly. Another reason for weakening the coatings and lowering the roughness is aggregation of $M B G N$ in the coating matrix. The aggregated MBGN in coatings prevent the strong bonding between other nanoparticles and also creates microporous structure. These microporous structure endow enough space to weaken the coating strength.

We tested the biological properties of the composite coating by immersing it into SBF solution. The MBGN-0.50 showed attracted increased deposition of spherical hydroxyapatite on the surface, probably owing to ion release from the MBGN and surface roughness. Surface roughness plays a key role in apatite formation from SBF. For example, it has been shown that the titanium implants with surface roughness ranging from 0.44 to $8.68 \mu \mathrm{m}$ elicit better osseointegration ${ }^{42}$. Moreover, nanoroughness can also influence the cellular activities such as adhesion migration, proliferation and differentiation. For example, as acid-etched titanium surface with average roughness $(\mathrm{Ra}) 49$ to $110 \mathrm{~nm}$ has shown positive influence on rat bone-marrow derived osteoblast differentiation ${ }^{43-44}$. The coating of MBGN with chitosan increased the surface roughness and led to increased apatite precipitation compare to pure chitosan. Finally, the size-aspect can also account for better cell adhesion as size of MBGN plays an important role in cellular activities. The spherical MBGN of size $90 \pm 10 \mathrm{~nm}$ give the nanotopographical cues that affect the cellular functions. Various physiological and pathological events occurs in the cells are directly associated with the nanotopography and shape and size of the nanomaterials ${ }^{45}$. In this study, MC3T3-E1 cells were spindle-shaped and elongated on MBGN-0.50 substrates compared to cells on Chi coatings because cells sense a nanotopographical surface which has embedded nanosized spherical MBGN. Although, integrins can behave different on the surfaces with roughness, Young's Modulus, and adhesive molecules ${ }^{46}$, more studies should be performed in the future to understand the integrin action. However, it is very difficult to explain how the integrin of the cell surface sense the nanosized spherical MBGN but it 
will be considerably different from the flat Chi coating surface. Moreover, stem cells on 70-100 nm nanotubes showed elongated morphology with increased stress fibers and differentiated to an osteogenic lineage ${ }^{47-48}$. We also found elongated morphology of the MC3T3-E1 cells on MBGN-0.50 substrates. We suspect the elongated morphology along with the increased stress fibers inside the cells led to osteogenic differentiation on MBG- 0.50 substrates owing to MBGN. The stiffness/nanoroughness of the coating also enhances the cell spreading and increased ALP activity of MC3T3-E1 cells ${ }^{42}$. The ions release data from coating revealed that the calcium ion level released was $\sim 248$ ppm for 21 days, which might play a key role in stimulating osteoblastic cellular events. It has been reported that the extracellular calcium ion promote proliferation, migration and osteogenic differentiation of cells ${ }^{49}$, and the effects of silicate ions on osteoblastic differentiation and angiogenesis have also been studies ${ }^{50-51}$. Finally, the q-RT-PCR results confirmed the role of MBGN to promote the osteogenic differentiation of MC3T3-E1 cells. The strong staining of OPN on MBGN-0.50 coating compare to Chi also confirmed the differentiation result. The coating is essentially an organic-inorganic matrix, with chitosan being the organic part and the MBGN being the inorganic part. Apart from the nanotopography, the cells also sense this stiff substrate, which is favorable for osteogenic differentiation. Thus, nanotopography, a stiff surface, and inorganic ions such as $\mathrm{Ca}$ and Si released from the MBGN contribute to the superior performance of MBGN-Chi nanocomposite material for osteogenic differentiation. We have studied the osteogenic differentiation of MC3T3-E1 cell which is a pre-osteoblast cell line on MBGN-0.5 and Chi coating. However, other studies like the effect of different nanoroughness and ions release for controlled and specific lineage differentiation will taken up in future..

\section{CONCLUSIONS}

Highly mesoporous bioactive glass nanoparticles (MBGN) with naturally derived biopolymer chitosan (Chi) are successfully utilized to produce bioactive nanotopographical composite coatings on titanium substrates by electro-deposition. The MBGN/Chi coating weight gain was well controlled by the electrodeposition parameters (time and voltage) at constant solution $\mathrm{pH}$ of 3.5. The apatite-forming ability of MBGN/Chi composite coatings was excellent in simulated body fluid. The mechanical strength and nanoroughness for MBGN-0.50 nanocomposite coatings was ideal with respect to mechanical strength 
and roughness and also exhibited suitable properties for cell adhesion, spreading, proliferation and ALP activity of the MC3T3-E1 cells. The subsequent osteoblastic differentiation behaviors on the engineered surface, as examined up to 21 days, showed significantly up-regulated expression of bone-associated genes (ALP, Col I, OPN, and OCN). These results indicate that the combinatory cues provided by nanotopology (25 $\mathrm{nm}$ roughness) and ions released from MBGN are highly effective in stimulating osteoblastic differentiation and that suggest the MBGN/chitosan may serve as a potential composition for bone implant coatings.

\section{ASSOCIATED CONTENT}

\section{Supporting Information}

Table S1. List of osteogenic primers, corresponding gene ID and forward \& reverse sequences.

Figure $\mathbf{S 1}$. $\xi$-potentials of mesoporous bioglass nanoparticles before and after amination.

Figure S2. Pore size distributions.

Figure S3. Mechanical strength and nanoroughness; (a) surface topography of MBGN-0.25 and (b) MBGN-0.75 coatings with $2 \mathrm{D}$ and $3 \mathrm{D}$ view before and after indentation; nanoindentation profile of indents for (c) MBGN-0.25, and (d) MBGN-0.75 at $3 \mathrm{mN}$ peak load.

\section{AUTHOR INFORMATION}

\section{Corresponding Author}

*kapildpatel20@gmail.com, kapil.patel@ucl.ac.uk

\section{Author Contributions}

The manuscript was written with contributions from all authors. All authors have given approval to the final version of the manuscript.

\section{Notes}

The authors declare no competing financial interest.

\section{ACKNOWLEDGMENTS}

This research was supported by the National Research Foundation (NRF) of Republic of Korea (Global Research Lab Program 2015-0093829, Global Research Development Center Program 2018R1D1A1B07048020, 2018K1A4A3A01064257, and 2017R1C1B1011387). 


\section{REFERENCES}

(1) Hatamleh, M. M.; Wu, X.; Alnazzawi, A.; Watson, J.; Watts, D. Surface characteristics and biocompatibility of cranioplasty titanium implants following different surface treatments. Dental Materials 2018, 34 (4), 676-683.

(2) Feng, X.; McDonald, J. M. Disorders of bone remodeling. Annu Rev Pathol 2011, 6, 121-145.

(3) Thoma, D. S.; Buranawat, B.; Hammerle, C. H.; Held, U.; Jung, R. E. Efficacy of soft tissue augmentation around dental implants and in partially edentulous areas: a systematic review. $J$ Clin Periodontol 2014, 41 Suppl 15, S77-S91.

(4) Shen, X.; Zhang, Y.; Ma, P.; Sutrisno, L.; Luo, Z.; Hu, Y.; Yu, Y.; Tao, B.; Li, C.; Cai, K. Fabrication of magnesium/zinc-metal organic framework on titanium implants to inhibit bacterial infection and promote bone regeneration. Biomaterials 2019, 212, 1-16.

(5) Sartoretto, S. C.; Alves, A. T.; Resende, R. F.; Calasans-Maia, J.; Granjeiro, J. M.; Calasans-Maia, M.

D. Early osseointegration driven by the surface chemistry and wettability of dental implants. $J$ Appl Oral Sci 2015, 23 (3), 279-287.

(6) Civantos, A.; Martínez-Campos, E.; Ramos, V.; Elvira, C.; Gallardo, A.; Abarrategi, A. Titanium Coatings and Surface Modifications: Toward Clinically Useful Bioactive Implants. ACS Biomaterials Science \& Engineering 2017, 3 (7), 1245-1261.

(7) Rupp, F.; Gittens, R. A.; Scheideler, L.; Marmur, A.; Boyan, B. D.; Schwartz, Z.; Geis-Gerstorfer, J. A review on the wettability of dental implant surfaces I: theoretical and experimental aspects. Acta biomaterialia 2014, 10 (7), 2894-2906.

(8) Huang, Y.-Z.; He, S.-K.; Guo, Z.-J.; Pi, J.-K.; Deng, L.; Dong, L.; Zhang, Y.; Su, B.; Da, L.-C.; Zhang, L.; Xiang, Z.; Ding, W.; Gong, M.; Xie, H.-Q. Nanostructured titanium surfaces fabricated by hydrothermal method: Influence of alkali conditions on the osteogenic performance of implants. Materials Science and Engineering: C 2019, 94, 1-10.

(9) Patel, K. D.; Kim, T.-H.; Lee, E.-J.; Han, C.-M.; Lee, J.-Y.; Singh, R. K.; Kim, H.-W. Nanostructured Biointerfacing of Metals with Carbon Nanotube/Chitosan Hybrids by Electrodeposition for Cell Stimulation and Therapeutics Delivery. ACS Applied Materials \& Interfaces 2014, 6 (22), 20214-20224.

(10) Ma, Y.; Han, J.; Wang, M.; Chen, X.; Jia, S. Electrophoretic deposition of graphene-based materials: A review of materials and their applications. Journal of Materiomics 2018, 4 (2), 108-120.

(11) Patel, K. D.; Singh, R. K.; Lee, J.-H.; Kim, H.-W. Electrophoretic coatings of hydroxyapatite with various nanocrystal shapes. Materials Letters 2019, 234, 148-154.

(12) Bräuer, G.; Kondruweit, S. Surface and coating technologies. In Technology Guide: Principles Applications - Trends; Bullinger, H.-J., Ed.; Springer Berlin Heidelberg: Berlin, Heidelberg, 2009; pp 4247.

(13) Chen, W.; Shao, Y.; Li, X.; Zhao, G.; Fu, J. Nanotopographical Surfaces for Stem Cell Fate Control: Engineering Mechanobiology from the Bottom. Nano Today 2014, 9 (6), 759-784.

(14) Patel, K. D.; Mahapatra, C.; Jin, G.-Z.; Singh, R. K.; Kim, H.-W. Biocompatible Mesoporous Nanotubular Structured Surface to Control Cell Behaviors and Deliver Bioactive Molecules. ACS Applied Materials \& Interfaces 2015, 7 (48), 26850-26859.

(15) Dalby, M. J.; Gadegaard, N.; Oreffo, R. O. Harnessing nanotopography and integrin-matrix interactions to influence stem cell fate. Nature materials 2014, 13 (6), 558-569.

(16) Wang, N.; Tytell, J. D.; Ingber, D. E. Mechanotransduction at a distance: mechanically coupling the extracellular matrix with the nucleus. Nature reviews. Molecular cell biology 2009, 10 (1), 75-82.

(17) Seo, S.-J.; Chen, M.; Wang, H.; Kang, M. S.; Leong, K. W.; Kim, H.-W. Extra- and intra-cellular fate of nanocarriers under dynamic interactions with biology. Nano Today 2017, 14, 84-99.

(18) Lord, M. S.; Foss, M.; Besenbacher, F. Influence of nanoscale surface topography on protein adsorption and cellular response. Nano Today 2010, 5 (1), 66-78.

(19) Wang, X.; Shah, F. A.; Palmquist, A.; Grandfield, K. 3D Characterization of Human Nanoosseointegration by On-Axis Electron Tomography without the Missing Wedge. ACS Biomaterials Science \& Engineering 2017, 3 (1), 49-55.

(20) Hwang, J.-H.; Lee, D.-H.; Byun, M. R.; Kim, A. R.; Kim, K. M.; Park, J. I.; Oh, H. T.; Hwang, E. S.; Lee, K. B.; Hong, J.-H. Nanotopological plate stimulates osteogenic differentiation through TAZ activation. Scientific Reports 2017, 7 (1), 3632. 
(21) Lee, J.-H.; El-Fiqi, A.; Mandakhbayar, N.; Lee, H.-H.; Kim, H.-W. Drug/ion co-delivery multi-functional nanocarrier to regenerate infected tissue defect. Biomaterials 2017, 142, 62-76.

(22) Hu, H.; Tang, Y.; Pang, L.; Lin, C.; Huang, W.; Wang, D.; Jia, W. Angiogenesis and Full-Thickness Wound Healing Efficiency of a Copper-Doped Borate Bioactive Glass/Poly(lactic-co-glycolic acid) Dressing Loaded with Vitamin E in Vivo and in Vitro. ACS Applied Materials \& Interfaces 2018, 10 (27), 22939-22950.

(23) Zheng, K.; Wu, J.; Li, W.; Dippold, D.; Wan, Y.; Boccaccini, A. R. Incorporation of Cu-Containing Bioactive Glass Nanoparticles in Gelatin-Coated Scaffolds Enhances Bioactivity and Osteogenic Activity. ACS Biomaterials Science \& Engineering 2018, 4 (5), 1546-1557.

(24) El-Fiqi, A.; Buitrago, J. O.; Yang, S. H.; Kim, H. W. Biomimetically grown apatite spheres from aggregated bioglass nanoparticles with ultrahigh porosity and surface area imply potential drug delivery and cell engineering applications. Acta Biomater 2017, 60, 38-49.

(25) El-Fiqi, A.; Kim, J. H.; Kim, H. W. Osteoinductive fibrous scaffolds of biopolymer/mesoporous bioactive glass nanocarriers with excellent bioactivity and long-term delivery of osteogenic drug. ACS Appl Mater Interfaces 2015, 7 (2), 1140-1152.

(26) Singh, R. K.; Patel, K. D.; Mahapatra, C.; Kang, M. S.; Kim, H.-W. C-Dot Generated Bioactive Organosilica Nanospheres in Theranostics: Multicolor Luminescent and Photothermal Properties Combined with Drug Delivery Capacity. ACS Applied Materials \& Interfaces 2016, 8 (37), 24433-24444.

(27) Fischer-Cripps, A. C. Critical review of analysis and interpretation of nanoindentation test data. Surface and Coatings Technology 2006, 200 (14), 4153-4165.

(28) El-Fiqi, A.; Kim, T.-H.; Kim, M.; Eltohamy, M.; Won, J.-E.; Lee, E.-J.; Kim, H.-W. Capacity of mesoporous bioactive glass nanoparticles to deliver therapeutic molecules. Nanoscale 2012, 4 (23), 7475-7488.

(29) Sarkar, P.; Nicholson, P. S. Electrophoretic Deposition (EPD): Mechanisms, Kinetics, and Application to Ceramics. Journal of the American Ceramic Society 1996, 79 (8), 1987-2002.

(30) Pranoto, Y.; Rakshit, S. K.; Salokhe, V. M. Enhancing antimicrobial activity of chitosan films by incorporating garlic oil, potassium sorbate and nisin. LWT - Food Science and Technology 2005, 38 (8), 859-865.

(31) Carson, L.; Kelly-Brown, C.; Stewart, M.; Oki, A.; Regisford, G.; Luo, Z.; Bakhmutov, V. I. Synthesis and characterization of chitosan-carbon nanotube composites. Materials letters 2009, 63 (6-7), 617-620.

(32) Patel, K. D.; El-Fiqi, A.; Lee, H.-Y.; Singh, R. K.; Kim, D.-A.; Lee, H.-H.; Kim, H.-W. Chitosannanobioactive glass electrophoretic coatings with bone regenerative and drug delivering potential. Journal of Materials Chemistry 2012, 22 (47), 24945-24956.

(33) Kang, M. S.; Kim, J.-H.; Singh, R. K.; Jang, J.-H.; Kim, H.-W. Therapeutic-designed electrospun bone scaffolds: Mesoporous bioactive nanocarriers in hollow fiber composites to sequentially deliver dual growth factors. Acta Biomaterialia 2015, 16, 103-116.

(34) Patil, S.; Sandberg, A.; Heckert, E.; Self, W.; Seal, S. Protein adsorption and cellular uptake of cerium oxide nanoparticles as a function of zeta potential. Biomaterials 2007, 28 (31), 4600-4607.

(35) Wood, M. A.; Yang, Y.; Baas, E.; Meredith, D. O.; Richards, R. G.; Kuiper, J. H.; El Haj, A. J. Correlating cell morphology and osteoid mineralization relative to strain profile for bone tissue engineering applications. J R Soc Interface 2008, 5 (25), 899-907.

(36) Curran, J. M.; Chen, R.; Hunt, J. A. Controlling the phenotype and function of mesenchymal stem cells in vitro by adhesion to silane-modified clean glass surfaces. Biomaterials 2005, 26 (34), 7057-7067.

(37) Lu, M.; Shao, D.; Wang, P.; Chen, D.; Zhang, Y.; Li, M.; Zhao, J.; Zhou, Y. Enhanced osteoblast adhesion on amino-functionalized titanium surfaces through combined plasma enhanced chemical vapor deposition (PECVD) method. RSC Advances 2016, 6 (86), 82688-82697.

(38) Zhou, Z.; Dai, Y.; Liu, B.-b.; Xia, L.-I.; Liu, H.-b.; Vadgama, P.; Liu, H.-r. Surface modification of titanium plate enhanced fibronectin-mediated adhesion and proliferation of MG-63 cells. Transactions of Nonferrous Metals Society of China 2014, 24 (4), 1065-1071.

(39) Wang, Y.; Lei, T.; Jiang, B.; Guo, L. Growth, microstructure and mechanical properties of microarc oxidation coatings on titanium alloy in phosphate-containing solution. Applied Surface Science 2004, 233 (1), 258-267.

(40) Gomez-Vega, J. M.; Saiz, E.; Tomsia, A. P.; Marshall, G. W.; Marshall, S. J. Bioactive glass coatings with hydroxyapatite and Bioglass $₫$ particles on Ti-based implants. 1. Processing. Biomaterials 2000, 21 (2), 105-111. 
(41) Facca, S.; Lahiri, D.; Fioretti, F.; Messadeq, N.; Mainard, D.; Benkirane-Jessel, N.; Agarwal, A. In Vivo Osseointegration of Nano-Designed Composite Coatings on Titanium Implants. ACS Nano 2011, 5 (6), 4790-4799.

(42) Guadarrama Bello, D.; Fouillen, A.; Badia, A.; Nanci, A. A nanoporous titanium surface promotes the maturation of focal adhesions and formation of filopodia with distinctive nanoscale protrusions by osteogenic cells. Acta Biomaterialia 2017, 60, 339-349.

(43) Takeuchi, K.; Saruwatari, L.; Nakamura, H. K.; Yang, J.-M.; Ogawa, T. Enhanced intrinsic biomechanical properties of osteoblastic mineralized tissue on roughened titanium surface. Journal of Biomedical Materials Research Part A 2005, 72A (3), 296-305.

(44) Kubo, K.; Att, W.; Yamada, M.; Ohmi, K.; Tsukimura, N.; Suzuki, T.; Maeda, H.; Ogawa, T. Microtopography of titanium suppresses osteoblastic differentiation but enhances chondroblastic differentiation of rat femoral periosteum-derived cells. Journal of Biomedical Materials Research Part $A$ 2008, 87A (2), 380-391.

(45) Kim, D.-H.; Provenzano, P. P.; Smith, C. L.; Levchenko, A. Matrix nanotopography as a regulator of cell function. The Journal of cell biology 2012, 197 (3), 351-360.

(46) Goreham, R. V.; Mierczynska, A.; Smith, L. E.; Sedev, R.; Vasilev, K. Small surface nanotopography encourages fibroblast and osteoblast cell adhesion. RSC Advances 2013, 3 (26), 10309-10317.

(47) Oh, S.; Brammer, K. S.; Li, Y. S. J.; Teng, D.; Engler, A. J.; Chien, S.; Jin, S. Stem cell fate dictated solely by altered nanotube dimension. Proceedings of the National Academy of Sciences 2009, 106 (7), 2130-2135.

(48) Changede, R.; Cai, H.; Wind, S. J.; Sheetz, M. P. Integrin nanoclusters can bridge thin matrix fibres to form cell-matrix adhesions. Nature Materials 2019, DOI: 10.1038/s41563-019-0460-y.

(49) Lee, M. N.; Hwang, H.-S.; Oh, S.-H.; Roshanzadeh, A.; Kim, J.-W.; Song, J. H.; Kim, E.-S.; Koh, J.-T. Elevated extracellular calcium ions promote proliferation and migration of mesenchymal stem cells via increasing osteopontin expression. Experimental \& Molecular Medicine 2018, 50 (11), 142.

(50) Kang, M. S.; Lee, N.-H.; Singh, R. K.; Mandakhbayar, N.; Perez, R. A.; Lee, J.-H.; Kim, H.-W. Nanocements produced from mesoporous bioactive glass nanoparticles. Biomaterials 2018, 162, 183199.

(51) Olmos Buitrago, J.; Perez, R. A.; El-Fiqi, A.; Singh, R. K.; Kim, J. H.; Kim, H. W. Core-shell fibrous stem cell carriers incorporating osteogenic nanoparticulate cues for bone tissue engineering. Acta Biomater 2015, 28, 183-192. 


\section{Supporting Information}

\section{Combined Effects of Nanoroughness and Ions Produced by Electrodeposition of Mesoporous Bioglass Nanoparticle for Bone Regeneration}

Kapil D. Patel ${ }^{1,2,4,5,{ }^{*}}$, Jennifer O. Buitrago ${ }^{1,2}$, S. Prakash Parthiban ${ }^{1}$, Jung-Hwan Lee ${ }^{1,2,3,4}$, Rajendra K. Singh ${ }^{1,2}$, Jonathan C. Knowles ${ }^{2,4,5,6}$, Hae-Won Kim ${ }^{1,2,3,4}$

${ }^{1}$ Institute of Tissue Regeneration Engineering (ITREN), Dankook University, Cheonan 31116, South Korea

2 Department of Nanobiomedical Science \& BK21 PLUS NBM Global Research Center for Regenerative Medicine, Dankook University, Cheonan 31116, South Korea

${ }^{3}$ Department of Biomaterials Science, School of Dentistry, Dankook University, Cheonan 31116, South Korea

4 UCL Eastman-Korea Dental Medicine Innovation Centre, Dankook University, Cheonan 31116, Republic of Korea

5 Division of Biomaterials and Tissue Engineering, Eastman Dental Institute, University College London, 256 Gray's Inn Road, London WC1X 8LD, United Kingdom

${ }^{6}$ The Discoveries Centre for Regenerative and Precision Medicine, UCL Campus, London, UK

*Corresponding author: Dr. K. D. Patel, tel: +82 41550 3081; fax: +82 415503085

E-mail: kapildpatel20@gmail.com, Kapil.patel@ucl.ac.uk

For: ACS Applied Bio Materials 
Table S1. List of osteogenic primers, corresponding gene ID and forward \& reverse sequences.

\begin{tabular}{llll}
\hline Gene name & Gene ID & FWD & REV \\
GAPDH & AF106860.2 & CCA TTC TTC CAC CTT TGA TG & CTG TTG CTG TAG CCA TAT TC \\
ALP & J03572.1 & GCT TCA AAC CTA GAC ACA AG & GGC TCA AAG AGA CCT AAG A \\
COL1A1 & BC133728.1 & CCT CAG AAG AAC TGG TAC AT & TCC ATA CTC GAA CTG GAA TC \\
OCN & M23637.1 & CCT CCT GGT TCA TTT CTT TG & CTG TCT CTC TGG TAG TTT GT \\
OPN & M99252.1 & CGC TTC TGT TCT TTC TGT G & TTG CTG TTC CTG TAA GTT TG \\
\hline
\end{tabular}




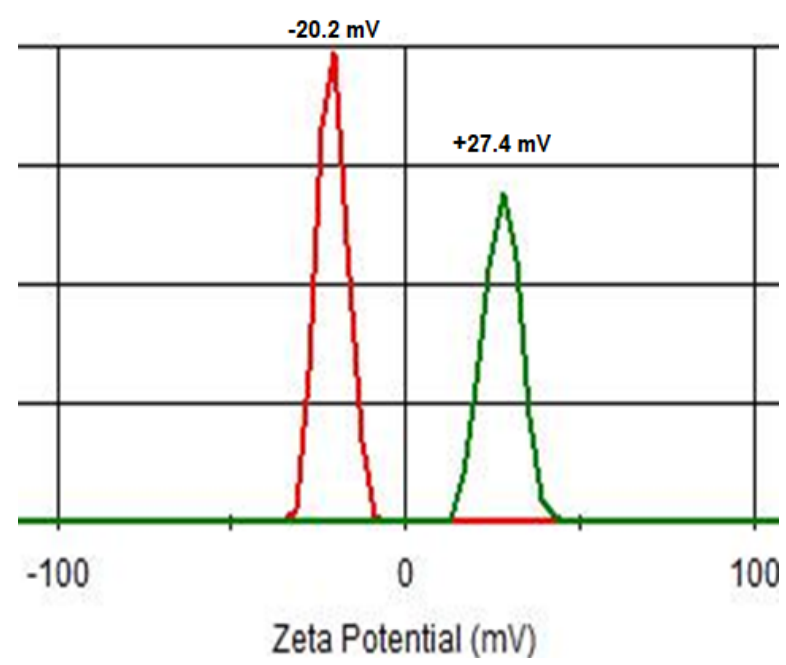

Figure $\mathbf{S} 1 . \xi$-potentials of mesoporous bioglass nanoparticles before and after amination. 


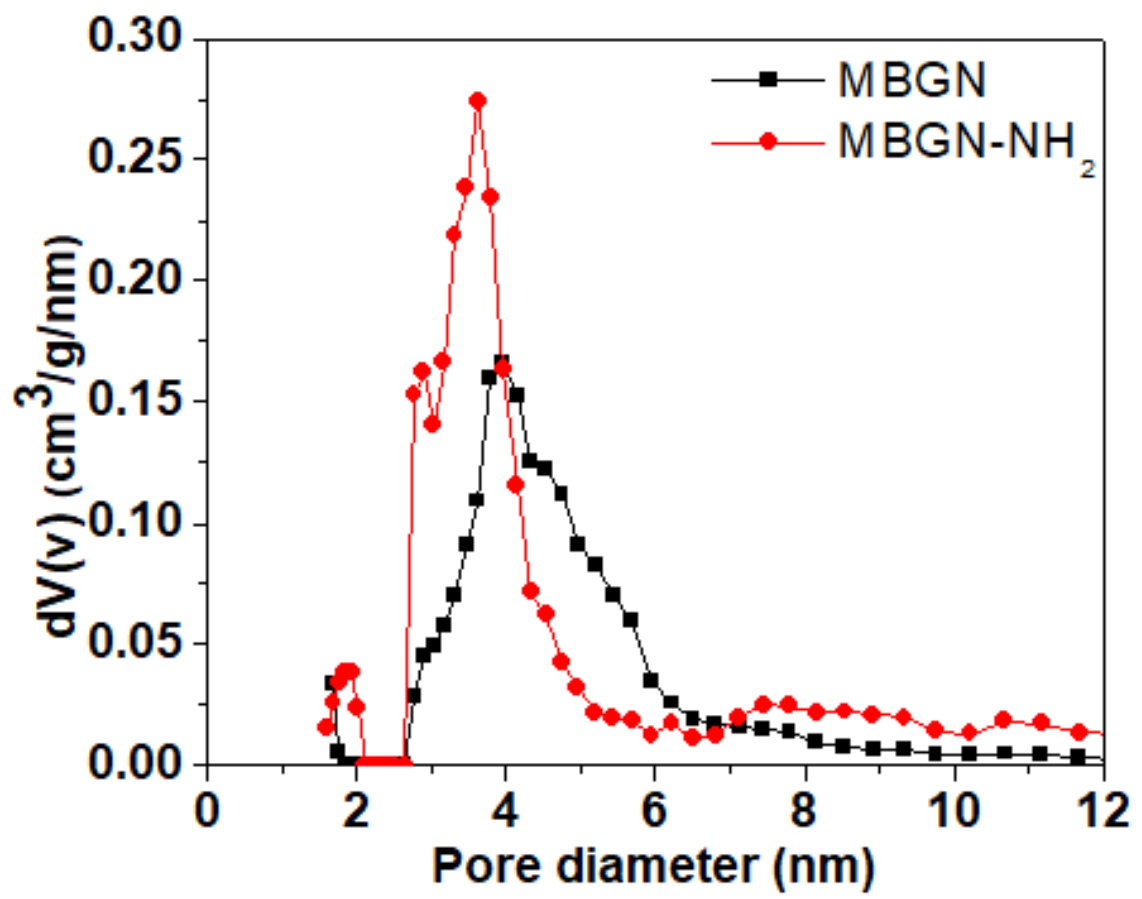

Figure S2. Pore size distributions. 

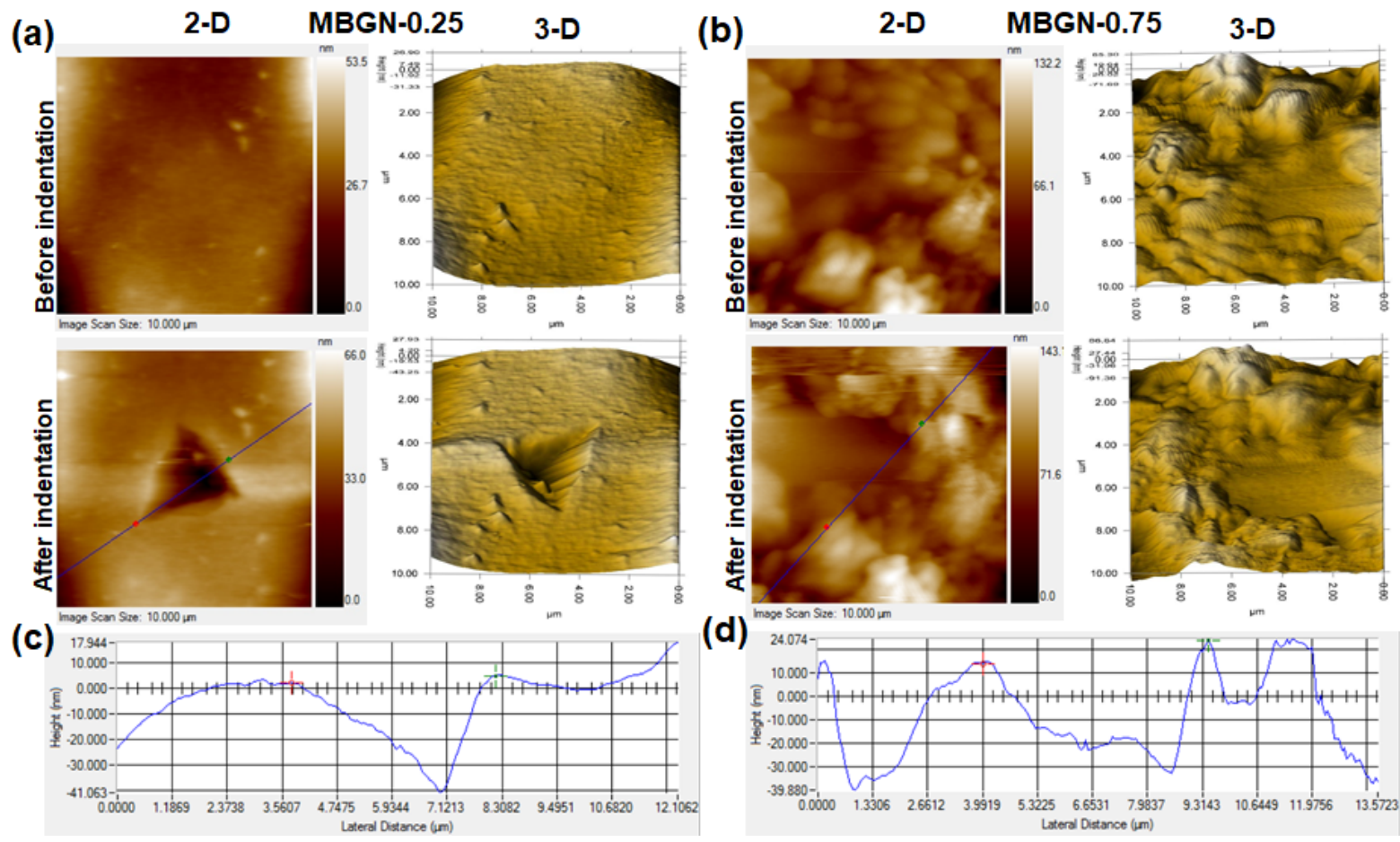

(d)

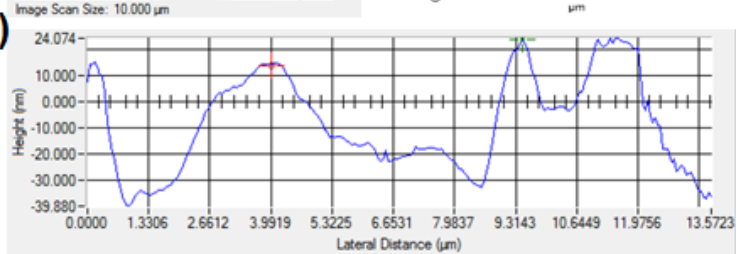

Figure S3. Mechanical strength and nanoroughness; (a) surface topography of MBGN-0.25 and (b) MBGN-0.75 coatings with 2D and 3D view before and after indentation; nanoindentation profile of indents for (c) MBGN-0.25, and (d) MBGN-0.75 at $3 \mathrm{mN}$ peak load. 